\title{
Natural Orbital Functional Theory
}

\author{
Mario Piris \\ Kimika Fakultatea, Euskal Herriko Unibertsitatea, and \\ Donostia International Physics Center (DIPC) \\ P.K. 1072, 20080 Donostia, Euskadi, Spain.
}

\subsection{INTRODUCTION}

In the last few years, the improvements in computer hardware and software have allowed the simulation of molecules and materials with an increasing number of atoms. However, the most accurate electronic structure methods based on $N$-particle wave functions, e.g., the configuration interaction (CI) method or the coupled cluster (CC) method, are computationally too expensive to be applied to large systems. It is clearer every day the need for treatments of electron correlation that scale favorably with the number of electrons.

As discussed in previous chapters of this book, since the interactions between electrons are pairwise within the Hamiltonian, the energy may be determined exactly from a knowledge of the two-particle reduced density matrix $(2-\mathrm{RDM}){ }^{2} \mathbf{D}$. The 2-RDM carries all the relevant information if one is interested in expectation values of one- and two-particle operators. In this manner, the $N$-particle dependence can be avoided given that the 2-RDM is a much more economic storage of information. There remains, however, the long-standing problem that not every 2-RDM is derivable from an $N$-particle wave function (the $N$-representability problem) [1]. As we have seen in last chapters, realistic variational 2-RDM calculations have recently become possible through the use of both the contracted Schrödinger equation and the optimization techniques known as semidefinite programming. Nevertheless, even the best first-order algorithms of semidefinite programming scale as $r^{6}$, where $r$ is the rank of the one-electron basis set.

In 1964, Hohenberg and Kohn (HK) [2] demonstrated that the ground state energy could be expressed as a functional of the one-electron density $\rho$ only. This result led to density functional theory (DFT) [3] which has 
become very popular thanks to its relatively low computational cost. Practical implementations of DFT are mainly based on the formulation of Kohn-Sham (KS) [4], in which the kinetic energy is not constructed as a functional of $\rho$ but rather from an auxiliary Slater determinant. Since the non-interacting kinetic energy differs from the many-body kinetic energy, there is a contribution from a part of the kinetic energy contained in the correlation potential. This correlation kinetic energy is the main source of problems of present-day KS functionals.

It seems that there is not $N$-representability problem since the conditions that ensure that a one-particle density comes from an $N$-particle wave function are well-known [5]. Here, the obstacle is the construction of the functional $E[\rho]$ capable of describing a quantum-mechanical $N$-electron system. This functional $N$-representability is still related to the $N$-representability problem of the 2-RDM. Many currently available functionals are not $N$-representable [6]. Consequently, the energies produced by these functionals can lie below the exact value. Even though these energy values may lie quite close to the exact ones they do not guarantee, however, that the calculations are accurate.

Another drawback of the most popular correlation functionals is that they exhibit an incorrect behavior for $N$-electron atoms as the nuclear charge $\mathrm{Z}$ increases [7]. Recently, the accuracy of DFT has been improved by using approximations constructed to satisfy exact constraints on the exchange-correlation energy functional [8]. Despite the great success achieved for high- $\mathrm{Z}$ atomic ions with density functionals of this new generation, they can not as yet adequately describe highly degenerate systems [9]. This corroborates the fact that KS functionals are better suited for describing dynamic correlation due to the short-range interelectronic repulsion than static correlation due to near degeneracy effects. The inability of approximate density functionals to account for the dispersion interactions constitutes a serious drawback. The density functional for the correlation kinetic energy remains unknown and how important is it for the dynamics of chemical reactions is an open question [9].

A direction for improving DFT lies in the development of a functional theory based upon the one-particle reduced density matrix (1-RDM) ${ }^{1} \mathbf{D}$ rather than on the one-electron density $\rho$. Like 2-RDM, the 1-RDM is a much simpler object than the $N$-particle wave function, but the ensemble $N$-representability conditions that have to be imposed on variations of ${ }^{1} \mathbf{D}$ are well-known [1]. The existence [10] and properties [11] of the total energy functional of the 1-RDM are well-established. Its development may be greatly aided by imposition of multiple constraints that are more strict and abundant than their DFT counterparts $[12,13]$.

The major advantage of a 1-RDM formulation is that the kinetic energy is explicitly defined and does not require the construction of a functional. The unknown functional in a ${ }^{1} \mathbf{D}$-based theory only needs to incorporate electron correlation. It does not rely on the concept of a fictitious non-interacting system. Consequently, ${ }^{1} \mathbf{D}$-scheme is not expected to suffer from the above mentioned limitations of KS methods. In fact, the correlation energy in 1- 
RDM theory scales homogeneously in contrast to the scaling properties of the correlation term in DFT [14]. Moreover, the 1-RDM completely determines the natural orbitals (NOs) and their occupation numbers (ONs). Accordingly, the ${ }^{1} \mathbf{D}$-functional incorporates fractional ONs in a natural way, which should provide a correct description of both dynamical and nondynamical correlation.

\subsection{GROUNDWORK TOWARDS A ${ }^{1}$ D-FUNCTIONAL THEORY}

The idea of a 1-RDM functional appeared some decades ago. At the commencement, the main effort was focused on the existence of this functional. In 1974, Gilbert proved the analogon of the Hohenberg-Kohn theorem for the 1-RDMs including nonlocal external potentials [15]. Berrondo and Goscinski [16] added a nonlocal external potential to the Hamiltonian and obtained a variational principle involving the $1-\mathrm{RDM}$ for a local external potential by eliminating the nonlocal external source. Donnelly and Parr [17] proved that the existence was already implied in the original HK theorem. They discussed extensively the properties of this energy functional and derived the Euler equations associated with the exact ground state [17, 18].

In 1979, an elegant proof of the existence was provided by Levy [10]. He demonstrated that the universal variational functional for the electronelectron repulsion energy of an $N$-representable trial 1-RDM can be obtained by searching all antisymmetric wave functions which yield a fixed ${ }^{1} \mathbf{D}$. It was shown that the functional does not require that a trial function for a variational calculation be associated with a ground state of some external potential. Thus, the v-representability is not required, only $N$-representability. As a result, the 1-RDM functional theories of preceding works were unified. A year later, Valone [19] extended the Levy's pure-state constrained-search to include all ensemble representable 1-RDMs. He demonstrated that no new constraints are needed in the occupation-number variation of the energy functional. Diverse constrained-search density functionals by Lieb [20, 21] also afforded insight into this issue. He proved independently that the constrained minimizations exist.

It is well-known that the exact electronic energy can also be given explicitly in terms of the spinless 1-RDM and the two-particle charge density (2-CD). This suggests an alternative viewpoint regarding ${ }^{1} \mathbf{D}$-functional theory. One could employ the exact functional but with an approximate 2-CD that is built from ${ }^{1} \mathbf{D}$ using a reconstruction functional ${ }^{2} \mathbf{D}\left[{ }^{1} \mathbf{D}\right]$. Perhaps, the first explicit approximate relation between ${ }^{2} \mathbf{D}$ and ${ }^{1} \mathbf{D}$ containing one free parameter was that proposed by Müller in 1984 [22]. The case where the parameter was set to $1 / 2$ was discussed years later by Buijse [23] who performed self-consistent calculations for the $\mathrm{H}_{2}$ molecule.

Zumbach and Maschke [24] discussed the ${ }^{1} \mathbf{D}$-functional using ensemble search. They derived the set of self-consistent equations which include the 
eigenvalues of the 1-RDM, and clarified the Gilbert's relation between these eigenvalues and the chemical potential. Ludeña et. al. [25] considered alternatively a variational procedure with built-in pure state $N$-representability conditions. The $N$-representability of the $1-\mathrm{RDM}$ was accomplished by taking into account the conditions for the mapping of the nth-order density operator into a given ${ }^{1} \mathbf{D}$. They arrived at the Valone's result: the problem of obtaining a pure-state $N$-representable 1-RDM requires only the known ensemble constraints, if the proper functional is known.

Levy identified the unknown part of the exact universal ${ }^{1} \mathbf{D}$-functional as the correlation energy $\mathrm{E}_{c}\left[{ }^{1} \mathbf{D}\right]$ and investigated a number of properties of $\mathrm{E}_{c}\left[{ }^{1} \mathbf{D}\right]$, including scaling, bounds, convexity, and asymptotic behavior [11]. He suggested approximate explicit forms for $\mathrm{E}_{c}\left[{ }^{1} \mathbf{D}\right]$ for computational purposes as well. Redondo presented a density-matrix formulation of several $a b$ initio methods [26]. His generalization of the HK theorem followed closely Levy's demonstration of a 1-RDM functional. López-Sandoval and Pastor [27] investigated the Levy's functional $\mathrm{E}_{c}\left[{ }^{1} \mathbf{D}\right]$ on lattice models.

Valdemoro [28] achieved a close approximation to the 2-RDM by using the anticommutating relation of fermion operators, or what is equivalent, the $N$-representability conditions. This work indicated that the development of 1-RDM functional theories should be couched in terms of explicitly antisymmetric reconstructions of the 2-RDM.

More recently, a renewed interest has appeared in the literature. An approximate exchange-correlation functional based on the diagonal elements of ${ }^{1} \mathbf{D}$ and $\left({ }^{1} \mathbf{D}\right)^{2}$ was proposed by Carlsson [29]. This functional was derived within tight-binding theory and used successfully in simple model calculations of several physical properties. Klein and Dreizler [30] developed a correlated 1-RDM theory with close connections to DFT perturbation theory. They derived formulas for first-order corrections to the Hartree-Fock (HF) 1-RDM and wrote down a formally exact expression for the correlation energy by using the linked-cluster expansion. Cioslowski and Lopez-Boada obtained by application of the hypervirial theorem an approximate functional of the HF 1-RDM in terms of three-electron integrals and an unknown screening function [31]. Their formalism incorporated dispersion effects and yielded two distinct asymptotics of the correlation energy of atoms and monoatomic ions at the limit of a large nuclear charge. Nooijen explored the possibility of using Green's functions and the extended Koopman's theorem (EKT) [32, 33] to arrive at a 1-RDM formulation [34].

The 1-RDM functional is called Natural Orbital Functional (NOF) when it is based upon the spectral expansion of ${ }^{1} \mathbf{D}$. The first parameter-free NOF constructed and tested on real physical systems is probably the one of Goedecker and Umrigar (GU) [35]. The basic form of this functional can be traced to Müller [22] but self-interaction corrected. The GU functional considering diagonal terms coincides with the Buijse and Baerends (BB) reconstruction [23], [36]. Optimizing typically $50 \mathrm{NOs}$ and ONs for a variety of atoms and ions it was found that the GU functional yielded energies and densities that were 
comparable or better than those from the generalized-gradient approximation (GGA) in the DFT. The GU functional does not suffer from dissociation problems. It satisfies the Hermiticity and particle permutation conditions but violates the non-negativity condition for the diagonal elements of the 2RDM. Moreover, the GU functional gives a wrong description of the ONs for the lower occupied levels and overestimates correlation effects in jellium at intermediate and high densities.

Following these first encouraging numerical results, several authors suggested new approximations to the 1-RDM functional. Holas [37], and Cioslowski and Pernal [38] proposed different generalizations of GU functional. These functionals were analyzed in detail from the perspective of the homogeneous electron gas (HEG) [39]. Csanyi and Arias (CA) [40] proposed another functional from the condition that the two-matrix is a tensor product of oneparticle operators and that it satisfies the Hermiticity and particle-permutation constraints. Unfortunately, the CA functional gives almost vanishing correlation energies in contrast to the GU functional. Expressions for the secondorder energy variations in the 1-RDM theory were derived, resulting in a formalism for time-independent response properties and stability conditions [41]. The derivatives of the electronic energy with respect to the number of electrons were found to be very sensitive to the used ${ }^{1} \mathbf{D}$-functional.

Another route to the construction of approximate 1-RDM functional involves employment of expressions for $E$ and ${ }^{1} \mathbf{D}$ afforded by some size-consistent formalism of electronic structure theory. Mazziotti [42] proposed a geminal functional theory (GFT) where an antisymmetric two-particle function (geminal) serves as the fundamental parameter. The one-matrix-geminal relationship allowed him to define a ${ }^{1} \mathbf{D}$-based theory from GFT [43]. He generalized the Levy's constrained search to optimize the universal functionals with respect to 2-RDMs rather than wave functions.

Csanyi and Arias [40] classified the above mentioned NOFs into two types: corrected Hartree $(\mathrm{CH})$ and corrected Hartree-Fock $(\mathrm{CHF})$. The performance of $\mathrm{CH}$ and $\mathrm{CHF}$ approximations in molecular calculations was investigated, taking molecules $\mathrm{H}_{2}, \mathrm{Li}_{2}$ and $\mathrm{LiH}$ as examples [44]. A ${ }^{1} \mathbf{D}$-based functional combining the properties of the $\mathrm{CH}$ and $\mathrm{CHF}$ approximations was also proposed [45]. An improved CHF-type functional leading to better results for the free-electron gas was suggested [46] too. The 2-CDs, intraculate and extraculate densities, $N$-representability and variational stability obtained with reconstruction functionals ${ }^{2} \mathbf{D}\left[{ }^{1} \mathbf{D}\right]$ that yield these ${ }^{1} \mathbf{D}$-based theories was investigated in detail [47].

The CA functional is similar to the Hartree-Fock-Bogoliubov (HFB) energy expression but differs by the sign of the post-HF correction term [48]. Existence of an HFB-type 1-RDM for systems with repulsive interactions was anticipated by other authors [49]. Different HFB-like functionals were proposed before for describing electronic structure [50]-[54].

Yasuda [55] obtained a correlation energy functional $\mathrm{E}_{c}\left[{ }^{1} \mathbf{D}\right]$ from the firstand second-order density equations together with the decoupling approxima- 
tions for the 3- and 4-reduced density matrices. The Yasuda functional is capable of properly describing a high-density HEG [56] and encouraging results were reported for atoms and molecules [55]. Some shortcomings of this functional were also pointed out [57].

Piris and Otto (PO) achieved a reconstruction functional ${ }^{2} \mathbf{D}\left[{ }^{1} \mathbf{D}\right]$ satisfying the most general properties of the 2-RDM [58]. They kept the spin structure from Ref. [52, 53], but introduced a new spatially dependence in the correction term of the 2-RDM. Calculated values for polarizabilities [59], ionization energies, equilibrium geometries, and vibrational frequencies [60] in molecules were obtained. An extension of this functional to periodic polymers was also considered [61]. In this line of constructing the 2-RDM from formal criteria, Kollmar and Hess [62] obtained an implicit functional where the elements of an idempotent matrix were used as variational parameters. The first application of the 1-RDM functional theory to open-shell molecular systems was presented using PO functional [60]. Recently, an open-shell formulation of the GU functional considering spin-dependent ONs was applied to the first-row atoms [63]. This approach conserves the $z$ component, but not the total spin. A study of the partitioning of the 1-RDM according to the theory of atoms in molecules has been reported too [64].

An extension of the antisymmetrized product of strongly orthogonal geminals (APSG) theory provided a "JK-only" expression for the electron-electron repulsion energy $\mathrm{V}_{e e}$ of a closed-shell system [65]. An implicit NOF involving pair-excitation coefficients instead of the ONs was proposed by Kollmar and Hess [66]. They considered a size-consistent extension of a limited multiconfiguration self-consistent field wave-function taking into account only pair excitations (PEMCSCF). In the case of four-electron systems, an approximate expression for $\mathrm{V}_{e e}$ was proposed [67] using a permanent-based parametrization of coefficients in a pair-excitation configuration interaction (CI) expansion. Moreover, a particular parametrization of coefficients in a CI expansion leaded to an explicit functional in terms of the Coulomb and exchange integrals over NOs, and an idempotent matrix, whose diagonal elements equal the ONs [68]. The thus obtained functional cannot, however, be employed in practical calculations due to the necessity to carry out minimizations over a large number of possible combinations of CI coefficient signs (phase dilemma). The size and volume extensivity of such functionals has been also analyzed by applying them to the HEG [69]. A JK-only 1-RDM functional starting from the PEMCSCF method and using necessary $N$-representability conditions for the 2-RDM has been recently derived [70]. This functional gives a small fraction of the total correlation energy for the water molecule at the equilibrium distance indicating that the accuracy of JK-only functional form may be limited. Nevertheless, two new JK-only approximations which recover a reasonable fraction of the total correlation energy at the equilibrium geometries have been recently proposed.

Gritsenko et. al. [71] have introduced several physical motivated repulsive corrections to the BB functional (BBC). With these corrections, they 
improved the quality of the $\mathrm{BB}$ potential energy curves for prototype fewelectron molecules and with BBC3 the average error of correlation energies for atomic systems at the equilibrium geometry was only $6 \%$. The ionization potentials produced by the $\mathrm{GU}$ and $\mathrm{BBC}$ functionals have been also investigated [72] using the EKT.

Piris [73] has recently proposed an explicit form for the cumulant [74, 75] of the 2-RDM in terms of two symmetric matrices, $\boldsymbol{\Delta}$ and $\boldsymbol{\Lambda}$. The suggested form of these matrices (as functions of the ONs) produces a NOF that reduces to the exact expression for the total energy in two-electron closed-shell systems $[76,77]$. One can generalize it to the $N$-electron systems, except for the off-diagonal elements of $\boldsymbol{\Delta}$. Alternatively, the mean value theorem and the partial sum rule for matrix $\boldsymbol{\Delta}$ provided a prescription for deriving a practical NOF. An assessment of this practical functional in molecules for calculating molecular properties, namely, polarizabilities [78], equilibrium bond distances, harmonic vibrational frequencies and vertical and adiabatic ionization potentials [79] has been performed. An extension of this functional to open-shell systems has been also considered [80].

We continue this chapter with a presentation of the basic concepts and notations relevant to ${ }^{1} \mathbf{D}$-functional theory (Section 3 ). We then review the fundaments of the NOF theory (Section 4) and derive the corresponding Euler equations (Section 5). The Gilbert [15] and Pernal [81] formulations, as well as the relation of Euler equations with the EKT are considered here. The following sections are devoted to present our NOF theory. The cumulant of the 2-RDM is discussed in detail in Section 6. The spin-restricted formulations for closed- and open-shells are analyzed in sections 7 and 8 , respectively. Section 9 is dedicated to our further simplification in order to achieve a practical functional. In section 10, we describe briefly how is implemented the NOF theory for numerical calculations. We end with some results for selected molecules (Section 11).

\subsection{1- AND 2-REDUCED DENSITY MATRICES}

The electronic energy $E$ for $N$-electron systems is an exactly and explicitly known functional of the 1- and 2-RDMs. The energy expression in spin-orbital (SO) representation is given by

$$
E=\sum_{i k}{ }^{1} D_{k}^{i} h_{i}^{k}+\sum_{i j k l}{ }^{2} D_{k l}^{i j}\langle k l \mid i j\rangle
$$

where $h_{i}^{k}$ are the one-electron matrix elements of the core-Hamiltonian,

$$
h_{i}^{k}=\int d \mathbf{x} \phi_{k}^{*}(\mathbf{x})\left[-\frac{1}{2} \nabla^{2}-\sum_{I} \frac{Z_{I}}{\left|\mathbf{r}-\mathbf{r}_{I}\right|}\right] \phi_{i}(\mathbf{x})
$$


viii

and $\langle k l \mid i j\rangle$ are the two-electron integrals of the Coulomb interaction,

$$
\langle k l \mid i j\rangle=\int d \mathbf{x}_{1} d \mathbf{x}_{2} \phi_{k}^{*}\left(\mathbf{x}_{1}\right) \phi_{l}^{*}\left(\mathbf{x}_{2}\right) r_{12}^{-1} \phi_{i}\left(\mathbf{x}_{1}\right) \phi_{j}\left(\mathbf{x}_{2}\right)
$$

Atomic units are used. Here and in the following $\mathbf{x} \equiv(\mathbf{r}, \mathbf{s})$ stands for the combined spatial and spin coordinates, $\mathbf{r}$ and $\mathbf{s}$, respectively. The SOs $\left\{\phi_{i}(\mathbf{x})\right\}$ constitute a complete orthonormal set of single-particle functions,

$$
\left\langle\phi_{k} \mid \phi_{i}\right\rangle=\int d \mathbf{x} \phi_{k}^{*}(\mathbf{x}) \phi_{i}(\mathbf{x})=\delta_{i}^{k}
$$

with an obvious meaning of the Kronecker delta $\delta_{i}^{k}$. Every normalizable function $\phi(\mathbf{x})$ of a single coordinate $\mathbf{x}$ may be expanded in the form

$$
\phi(\mathbf{x})=\sum_{i} \phi_{i}(\mathbf{x}) c_{i}, \quad c_{i}=\int d \mathbf{x} \phi_{i}^{*}(\mathbf{x}) \phi(\mathbf{x})
$$

We employ Löwdin's normalization convention [82] in which the trace of the 1-RDM equals the number of electrons

$$
\operatorname{Tr}{ }^{1} \mathbf{D}=\sum_{i}{ }^{1} \mathbf{D}_{i}^{i}=N
$$

and the trace of the 2-RDM gives the number of electron pairs in the system

$$
\operatorname{Tr}{ }^{2} \mathbf{D}=\sum_{i k}{ }^{2} D_{k k}^{i i}=\frac{N(N-1)}{2}=\left(\begin{array}{c}
N \\
2
\end{array}\right)
$$

The 1- and 2-RDMs can be obtained in the coordinate-space representation via the expansion theorem:

$$
\begin{gathered}
{ }^{1} D\left(\mathbf{x}_{1}^{\prime} \mid \mathbf{x}_{1}\right)=\sum_{i k}{ }^{1} D_{k}^{i} \phi_{i}^{*}\left(\mathbf{x}_{1}^{\prime}\right) \phi_{k}\left(\mathbf{x}_{1}\right) \\
{ }^{2} D\left(\mathbf{x}_{1}^{\prime}, \mathbf{x}_{2}^{\prime} \mid \mathbf{x}_{1}, \mathbf{x}_{2}\right)=\sum_{i j k l}{ }^{2} D_{k l}^{i j} \phi_{i}^{*}\left(\mathbf{x}_{1}^{\prime}\right) \phi_{j}^{*}\left(\mathbf{x}_{2}^{\prime}\right) \phi_{k}\left(\mathbf{x}_{1}\right) \phi_{l}\left(\mathbf{x}_{2}\right)
\end{gathered}
$$

The diagonal elements of ${ }^{1} \mathbf{D}$ and ${ }^{2} \mathbf{D}$ are always nonnegative, since ${ }^{1} D\left(\mathbf{x}_{1} \mid \mathbf{x}_{1}\right)$ is related to the probability of finding one electron at $\mathbf{x}_{1}$, and ${ }^{2} D\left(\mathbf{x}_{1}, \mathbf{x}_{2} \mid \mathbf{x}_{1}, \mathbf{x}_{2}\right)$ is related to the probability of finding one electron at $\mathbf{x}_{1}$ and another at $\mathbf{x}_{2}$. The diagonal elements ${ }^{1} D_{i}^{i}$ and ${ }^{2} D_{k k}^{i i}$ may be interpreted analogously: ${ }^{1} D_{i}^{i}$ is related to the probability of finding one electron in the spin-orbital $i$ when all the other electrons occupy arbitrary spin-orbitals, ${ }^{2} D_{k k}^{i i}$ is related to the probability of finding one electron in the spin-orbital $i$ and another in the spin-orbital $k$, when all other particles may occupy arbitrary spin-orbitals. 
As discussed in preceding chapters of this book, the 2-RDM is Hermitian

$$
{ }^{2} D\left(\mathbf{x}_{1}^{\prime}, \mathbf{x}_{2}^{\prime} \mid \mathbf{x}_{1}, \mathbf{x}_{2}\right)={ }^{2} D^{*}\left(\mathbf{x}_{1}, \mathbf{x}_{2} \mid \mathbf{x}_{1}^{\prime}, \mathbf{x}_{2}^{\prime}\right) \quad\left[{ }^{2} D_{k l}^{i j}=\left({ }^{2} D_{i j}^{k l}\right)^{*}\right]
$$

It is antisymmetric in each set of indices

$$
\begin{array}{ll}
{ }^{2} D\left(\mathbf{x}_{1}^{\prime}, \mathbf{x}_{2}^{\prime} \mid \mathbf{x}_{1}, \mathbf{x}_{2}\right)=-{ }^{2} D\left(\mathbf{x}_{1}^{\prime}, \mathbf{x}_{2}^{\prime} \mid \mathbf{x}_{2}, \mathbf{x}_{1}\right) & \left({ }^{2} D_{k l}^{i j}=-{ }^{2} D_{l k}^{i j}\right) \\
{ }^{2} D\left(\mathbf{x}_{1}^{\prime}, \mathbf{x}_{2}^{\prime} \mid \mathbf{x}_{1}, \mathbf{x}_{2}\right)=-{ }^{2} D\left(\mathbf{x}_{2}^{\prime}, \mathbf{x}_{1}^{\prime} \mid \mathbf{x}_{1}, \mathbf{x}_{2}\right) & \left({ }^{2} D_{k l}^{i j}=-{ }^{2} D_{k l}^{j i}\right)
\end{array}
$$

and symmetric with respect to particle permutation

$$
{ }^{2} D\left(\mathbf{x}_{1}^{\prime}, \mathbf{x}_{2}^{\prime} \mid \mathbf{x}_{1}, \mathbf{x}_{2}\right)={ }^{2} D\left(\mathbf{x}_{2}^{\prime}, \mathbf{x}_{1}^{\prime} \mid \mathbf{x}_{2}, \mathbf{x}_{1}\right) \quad\left({ }^{2} D_{k l}^{i j}={ }^{2} D_{l k}^{j i}\right)
$$

There is an important contraction relation between 1- and 2-RDMs that is in agreement with the previous normalization,

$$
{ }^{1} D\left(\mathbf{x}_{1}^{\prime} \mid \mathbf{x}_{1}\right)=\frac{2}{N-1} \int d \mathbf{x}^{2} D\left(\mathbf{x}_{1}^{\prime}, \mathbf{x} \mid \mathbf{x}_{1}, \mathbf{x}\right) \quad\left({ }^{1} D_{k}^{i}=\frac{2}{N-1} \sum_{j}{ }^{2} D_{k j}^{i j}\right)
$$

This implies that the energy functional (0.1) is just of the 2-RDM, because ${ }^{2} \mathbf{D}$ determines ${ }^{1} \mathbf{D}$. However, attempts to determine the energy by minimizing $E\left[{ }^{2} \mathbf{D}\right]$ are complicated due to the lack of a simple set of necessary and sufficient conditions for ensuring that the two-matrix corresponds to an $N$-particle wave function (the $N$-representability problem) [1]. Nevertheless, some necessary conditions have been derived (see Chapter 9). The so called D-condition is equivalent to the requirement that the 2 -RDM be positive semidefinite $\left({ }^{2} \mathbf{D} \geq 0\right)$. This constraint prevents the probability distribution for finding two particles in two SOs from being anywhere negative. The G- and Q-conditions state that the electron-hole density matrix $\mathbf{G}$ and the two-hole density matrix $\mathbf{Q}$ must be positive semidefinite too. The constraint $\mathbf{G} \geq 0(\mathbf{Q} \geq 0)$ enforces likewise this nonnegativity for a particle and a hole (two holes).

\subsubsection{Spin structure}

The $N$-electron Hamiltonian $\widehat{\mathcal{H}}$ corresponding to the expectation value $(0.1)$ does not contain any spin coordinates hence both operators $\widehat{S}_{z}$ and $\widehat{S}^{2}$ commute with $\widehat{\mathcal{H}}$. Consequently, the eigenfunctions of the Hamiltonian are also eigenfunctions of these two spin operators. In particular, according to Löwdin's expressions [82], we have

$$
\left\langle\widehat{S}_{z}\right\rangle=\int d \mathbf{x}_{1} \widehat{S}_{z}^{1} D\left(\mathbf{r}_{1} \mathbf{s}_{1} \mid \mathbf{r}_{1} \mathbf{s}_{1}\right)=M_{S}
$$




$$
\left\langle\widehat{S}^{2}\right\rangle=-\frac{N(N-4)}{4}+\int d \mathbf{x}_{1} d \mathbf{x}_{2}{ }^{2} D\left(\mathbf{r}_{1} \mathbf{s}_{1}, \mathbf{r}_{2} \mathbf{s}_{2} \mid \mathbf{r}_{1} \mathbf{s}_{2}, \mathbf{r}_{2} \mathbf{s}_{1}\right)=S(S+1)
$$

where $M_{S}$ and $S$ are the spin quantum numbers describing the $z$ component and the total spin of an $N$-electron eigenstate. A state with total spin $S$ has multiplicity $(2 S+1)$.

The SOs are direct products $\left|\phi_{i}\right\rangle=\left|\varphi_{p}\right\rangle \otimes|\sigma\rangle$, so the set of SOs $\left\{\phi_{i}(\mathbf{x})\right\}$ may be split into two subsets: $\left\{\varphi_{p}^{\alpha}(\mathbf{r}) \alpha(\mathbf{s})\right\}$ and $\left\{\varphi_{p}^{\beta}(\mathbf{r}) \beta(\mathbf{s})\right\}$. Given a set of $2 R$ spin-orbitals $\left\{\phi_{i} \mid i=1, \ldots, 2 R\right\}$, we have two sets of $R$ orthonormal spatial functions, $\left\{\varphi_{p}^{\alpha}(\mathbf{r})\right\}$ and $\left\{\varphi_{p}^{\beta}(\mathbf{r})\right\}$, such that in general the first set is not orthogonal to the second one. Nevertheless, the original set

$$
\begin{aligned}
\phi_{2 p-1}(\mathbf{x}) & =\varphi_{p}^{\alpha}(\mathbf{r}) \alpha(\mathbf{s}), & & p=1, \ldots, R \\
\phi_{2 p}(\mathbf{x}) & =\varphi_{p}^{\beta}(\mathbf{r}) \beta(\mathbf{s}), & & p=1, \ldots, R
\end{aligned}
$$

continues being orthonormal via the orthogonality of the spin functions

$$
\int d \mathbf{s} \alpha^{*}(\mathbf{s}) \beta(\mathbf{s})=\int d \mathbf{s} \beta^{*}(\mathbf{s}) \alpha(\mathbf{s})=0
$$

For $\widehat{S}_{z}$ eigenstates, only density matrix blocks that conserve the number of each spin type are nonvanishing. It is easily seen that two components of the 1-RDM, namely, ${ }^{1} \mathbf{D}_{\beta}^{\alpha}$ and ${ }^{1} \mathbf{D}_{\alpha}^{\beta}$, must vanish. One obtains

$$
{ }^{1} D\left(\mathbf{x}_{1}^{\prime} \mid \mathbf{x}_{1}\right)={ }^{1} D_{\alpha}^{\alpha}\left(\mathbf{r}_{1}^{\prime} \mid \mathbf{r}_{1}\right) \alpha^{*}\left(\mathbf{s}_{1}^{\prime}\right) \alpha\left(\mathbf{s}_{1}\right)+{ }^{1} D_{\beta}^{\beta}\left(\mathbf{r}_{1}^{\prime} \mid \mathbf{r}_{1}\right) \beta^{*}\left(\mathbf{s}_{1}^{\prime}\right) \beta\left(\mathbf{s}_{1}\right)
$$

From Eq. (0.15), considering the normalization condition for the 1-RDM (0.6), it then follows that

$$
\left\langle\widehat{S}_{z}\right\rangle=\int d \mathbf{r}_{1} Q_{z}\left(\mathbf{r}_{1} \mid \mathbf{r}_{1}\right)=\frac{N^{\alpha}-N^{\beta}}{2}=M_{S}
$$

where $N^{\sigma}$ is the number of electrons with $\sigma$ spin and $Q_{z}\left(\mathbf{r}_{1} \mid \mathbf{r}_{1}\right)$ represents the spin density [83],

$$
Q_{z}\left(\mathbf{r}_{1} \mid \mathbf{r}_{1}\right)=\frac{1}{2}\left[{ }^{1} D_{\alpha}^{\alpha}\left(\mathbf{r}_{1} \mid \mathbf{r}_{1}\right)-{ }^{1} D_{\beta}^{\beta}\left(\mathbf{r}_{1} \mid \mathbf{r}_{1}\right)\right]
$$

On the other hand, the 2-RDM has generally 16 spin blocks. As a result of the requirement $m_{s}(1)+m_{s}(2)=m_{s}\left(1^{\prime}\right)+m_{s}\left(2^{\prime}\right)$ for $\widehat{S}_{z}$ eigenstates, only 6 spin components are non-zero. Expanding the 2-RDM by spin components, we have

$$
\begin{gathered}
{ }^{2} D\left(\mathbf{x}_{1}^{\prime}, \mathbf{x}_{2}^{\prime} \mid \mathbf{x}_{1}, \mathbf{x}_{2}\right)=\left({ }^{2} \mathbf{D}_{\alpha \alpha}^{\alpha \alpha}\right) \alpha^{*} \alpha^{*} \alpha \alpha+\left({ }^{2} \mathbf{D}_{\alpha \beta}^{\alpha \beta}\right) \alpha^{*} \beta^{*} \alpha \beta+\left({ }^{2} \mathbf{D}_{\beta \alpha}^{\beta \alpha}\right) \beta^{*} \alpha^{*} \beta \alpha \\
+\left({ }^{2} \mathbf{D}_{\beta \alpha}^{\alpha \beta}\right) \alpha^{*} \beta^{*} \beta \alpha+\left({ }^{2} \mathbf{D}_{\alpha \beta}^{\beta \alpha}\right) \beta^{*} \alpha^{*} \alpha \beta+\left({ }^{2} \mathbf{D}_{\beta \beta}^{\beta \beta}\right) \beta^{*} \beta^{*} \beta \beta
\end{gathered}
$$


In fact only three of these components are independent, for example [see Eq. (0.11)],

$$
{ }^{2} D_{\alpha \beta}^{\alpha \beta}\left(\mathbf{r}_{1}^{\prime}, \mathbf{r}_{2}^{\prime} \mid \mathbf{r}_{1}, \mathbf{r}_{2}\right)=-{ }^{2} D_{\beta \alpha}^{\alpha \beta}\left(\mathbf{r}_{1}^{\prime}, \mathbf{r}_{2}^{\prime} \mid \mathbf{r}_{2}, \mathbf{r}_{1}\right) \quad\left({ }^{2} D_{r \alpha, t \beta}^{p \alpha, q \beta}=-{ }^{2} D_{t \beta, r \alpha}^{p \alpha, q \beta}\right)
$$

We may take the independent components to be ${ }^{2} \mathbf{D}_{\alpha \alpha}^{\alpha \alpha},{ }^{2} \mathbf{D}_{\alpha \beta}^{\alpha \beta},{ }^{2} \mathbf{D}_{\beta \beta}^{\beta \beta}$. The parallel-spin components must be antisymmetric, but ${ }^{2} \mathbf{D}_{\alpha \beta}^{\alpha \beta}$ possess no special symmetry. Each of these two-matrix blocks must contract to the appropriate one-matrix block, namely

$$
\begin{gathered}
\sum_{q}{ }^{2} D_{r \sigma, q \sigma}^{p \sigma, q \sigma}=\frac{\left(N^{\sigma}-1\right)}{2}{ }^{1} D_{r \sigma}^{p \sigma} \\
\sum_{q}{ }^{2} D_{r \alpha, q \beta}^{p \alpha, q \beta}=\frac{N^{\beta}}{2}{ }^{1} D_{r \alpha}^{p \alpha}
\end{gathered}
$$

It is readily demonstrated that the sum rules (0.24) and (0.25) are compatible with the Eq. (0.14). The traces of these two-matrix components read

$$
\operatorname{Tr}{ }^{2} \mathbf{D}_{\sigma \sigma}^{\sigma \sigma}=\frac{N^{\sigma}\left(N^{\sigma}-1\right)}{2}, \quad \operatorname{Tr}{ }^{2} \mathbf{D}_{\alpha \beta}^{\alpha \beta}=\frac{N^{\alpha} N^{\beta}}{2}
$$

From Eqs. (0.16) and (0.22), taking into account the orthonormality conditions (0.4) for each spin type, one obtains

$$
\left\langle\widehat{S}^{2}\right\rangle=-\frac{N(N-4)}{4}+\sum_{p q}\left({ }^{2} D_{p \alpha, q \alpha}^{p \alpha, q \alpha}+{ }^{2} D_{p \beta, q \beta}^{p \beta, q \beta}\right)-2 \sum_{p q r t}{ }^{2} D_{r \alpha, t \beta}^{p \alpha, q \beta} S_{t \beta}^{p \alpha} S_{r \alpha}^{q \beta}
$$

where $S_{t \sigma^{\prime}}^{p \sigma}=\left\langle\varphi_{p}^{\sigma} \mid \varphi_{t}^{\sigma^{\prime}}\right\rangle$ is the overlap matrix.

In this chapter, we consider later spin-polarized systems. One avenue of approach is to apply the spin unrestricted formalism where SOs have different spatial orbitals for different spins. However, this procedure can introduce important spin contamination effects through the last term of Eq. (0.27) since the overlap matrix $S_{t \beta}^{p \alpha} \neq \delta_{t}^{p}$. These effects can be avoided by the use of spin restricted theory. In this case only a single set of orbitals is used for $\alpha$ and $\beta$ spins

$$
\varphi_{p}^{\alpha}(\mathbf{r})=\varphi_{p}^{\beta}(\mathbf{r})=\varphi_{p}(\mathbf{r})
$$

The orthonormality requirement $\left(S_{t \beta}^{p \alpha}=\delta_{t}^{p}\right)$ leads to the expectation value of the total spin

$$
\left\langle\widehat{S}^{2}\right\rangle=-\frac{N(N-4)}{4}+\sum_{p q}\left({ }^{2} D_{p \alpha, q \alpha}^{p \alpha, q \alpha}+{ }^{2} D_{p \beta, q \beta}^{p \beta, q \beta}-2{ }^{2} D_{q \alpha, p \beta}^{p \alpha, q \beta}\right)
$$


By combining Eq. (0.1) with Eqs. (0.19) and (0.22), one arrives at the energy expression

$$
\begin{aligned}
E & =\sum_{p r} h_{p}^{r}\left({ }^{1} D_{r \alpha}^{p \alpha}+{ }^{1} D_{r \beta}^{p \beta}\right) \\
& +\sum_{p q r t}\langle r t \mid p q\rangle\left({ }^{2} D_{r \alpha, t \alpha}^{p \alpha, q \alpha}+{ }^{2} D_{r \alpha, t \beta}^{p \alpha, q \beta}+{ }^{2} D_{t \alpha, r \beta}^{q \alpha, p \beta}+{ }^{2} D_{r \beta, t \beta}^{p \beta, q \beta}\right)
\end{aligned}
$$

We must note that the two non-zero blocks ${ }^{2} D_{r \beta, t \alpha}^{p \alpha, q \beta}$ and ${ }^{2} D_{r \alpha, t \beta}^{p \beta, q \alpha}$ do not contribute to Eq. (0.30) since the corresponding two-electron matrix elements of the Coulomb interaction vanish. It is convenient now to introduce spinless density matrices [83]:

$$
\begin{gathered}
{ }^{1} \widetilde{D}_{r}^{p}=\sum_{\sigma}{ }^{1} D_{r \sigma}^{p \sigma}={ }^{1} D_{r \alpha}^{p \alpha}+{ }^{1} D_{r \beta}^{p \beta} \\
{ }^{2} \widetilde{D}_{r t}^{p q}={ }^{2} D_{r \alpha, t \alpha}^{p \alpha, q \alpha}+{ }^{2} D_{r \alpha, t \beta}^{p \alpha, q \beta}+{ }^{2} D_{r \beta, t \alpha}^{p \beta, q \alpha}+{ }^{2} D_{r \beta, t \beta}^{p \beta, q \beta}
\end{gathered}
$$

or in the coordinate-space representation

$$
\begin{gathered}
{ }^{1} \widetilde{D}\left(\mathbf{r}_{1}^{\prime} \mid \mathbf{r}_{1}\right)=\int d \mathbf{s}_{1}{ }^{1} D\left(\mathbf{r}_{1}^{\prime} \mathbf{s}_{1} \mid \mathbf{r}_{1} \mathbf{s}_{1}\right)=\sum_{\sigma}{ }^{1} D_{\sigma}^{\sigma}\left(\mathbf{r}_{1}^{\prime} \mid \mathbf{r}_{1}\right) \\
{ }^{2} \widetilde{D}\left(\mathbf{r}_{1}^{\prime} \mathbf{r}_{2}^{\prime} \mid \mathbf{r}_{1} \mathbf{r}_{2}\right)=\int d \mathbf{s}_{1} d \mathbf{s}_{2}{ }^{2} D\left(\mathbf{r}_{1}^{\prime} \mathbf{s}_{1}, \mathbf{r}_{2}^{\prime} \mathbf{s}_{2} \mid \mathbf{r}_{1} \mathbf{s}_{1}, \mathbf{r}_{2} \mathbf{s}_{2}\right) \\
=\sum_{\sigma \sigma^{\prime}}{ }^{2} D_{\sigma \sigma^{\prime}}^{\sigma \sigma^{\prime}}\left(\mathbf{r}_{1}^{\prime} \mathbf{r}_{2}^{\prime} \mid \mathbf{r}_{1} \mathbf{r}_{2}\right)
\end{gathered}
$$

The diagonal elements ${ }^{1} \widetilde{D}\left(\mathbf{r}_{1} \mid \mathbf{r}_{1}\right)$ and ${ }^{2} \widetilde{D}\left(\mathbf{r}_{1} \mathbf{r}_{2} \mid \mathbf{r}_{1} \mathbf{r}_{2}\right)$ are the electron density and pair density, respectively. The Eq. (0.30) can be rewritten as

$$
E=\sum_{p r}{ }^{1} \widetilde{D}_{r}^{p} h_{p}^{r}+\sum_{p q r t}{ }^{2} \widetilde{D}_{r t}^{p q}\langle r t \mid p q\rangle
$$

\subsection{THE NATURAL ORBITAL FUNCTIONAL (NOF)}

Let us replace the last term in Eq. (0.1), which is an explicit functional of the 2-RDM, by an unknown functional of the 1-RDM:

$$
E\left[N,{ }^{1} \mathbf{D}\right]=h\left[N,{ }^{1} \mathbf{D}\right]+V_{e e}\left[N,{ }^{1} \mathbf{D}\right]
$$


$h\left[N,{ }^{1} \mathbf{D}\right]$ is the contribution from the kinetic energy and the external potential,

$$
h\left[N,{ }^{1} \mathbf{D}\right]=\sum_{i k}{ }^{1} D_{k}^{i} h_{i}^{k}
$$

while the electron-electron repulsion energy $V_{e e}\left[N,{ }^{1} \mathbf{D}\right]$ constitutes an universal functional in the sense that it is invariant from one molecule to another for a given $N$, and hence it is independent of the external field. The constrainedsearch formalism [43] provides a proof by construction of the existence of this functional, in other words, it is given by the expression

$$
V_{e e}\left[N,{ }^{1} \mathbf{D}\right]=\min _{{ }^{2} \mathbf{D} \in \in^{2} \mathbf{D}\left({ }^{1} \mathbf{D}\right)} U\left[N,{ }^{2} \mathbf{D}\right]
$$

where

$$
U\left[N,{ }^{2} \mathbf{D}\right]=\int d \mathbf{x}_{1} d \mathbf{x}_{2}{ }^{2} D\left(\mathbf{x}_{1}, \mathbf{x}_{2} \mid \mathbf{x}_{1}, \mathbf{x}_{2}\right) r_{12}^{-1}=\sum_{i j k l}{ }^{2} D_{k l}^{i j}\langle k l \mid i j\rangle
$$

The notation ${ }^{2} \mathbf{D}\left({ }^{1} \mathbf{D}\right)$ indicates the family of 2 -RDMs which contract to the 1-RDM in agreement with Eq. (0.14). Restricting the 2-RDM in Eq. $(0.38)$ to be pure or ensemble $N$-representable yields the universal functionals of Levy [10] and Valone [19], respectively.

The properties of the universal functional $V_{e e}$ are well-known $[11,12,13]$. Moreover, the exact 1-RDM functional for the two-electron closed-shell systems like $\mathrm{H}_{2}$ or He is known too [76, 77]. However, $V_{e e}$ is highly difficult to approximate because what we have done is just to change the variational unknown from the 2-RDM to the 1-RDM, but the 2-RDM $N$-representability problems remain as reveals explicitly Eq. (0.38).

The 1-RDM can be diagonalized by a unitary transformation of the spinorbitals $\left\{\phi_{i}(\mathbf{x})\right\}$ with the eigenvectors being the natural spin-orbitals (NSOs) and the eigenvalues $\left\{n_{i}\right\}$ representing the ONs of the latter,

$$
{ }^{1} D_{k}^{i}=n_{i} \delta_{k}^{i}, \quad{ }^{1} D\left(\mathbf{x}_{1}^{\prime} \mid \mathbf{x}_{1}\right)=\sum_{i} n_{i} \phi_{i}^{*}\left(\mathbf{x}_{1}^{\prime}\right) \phi_{i}\left(\mathbf{x}_{1}\right)
$$

Restriction of the ONs $\left\{n_{i}\right\}$ to the range $0 \leq n_{i} \leq 1$ represents a necessary and sufficient condition for $N$-representability of the 1-RDM [1]. In the following, all representations used are assumed to refer to the basis of NSOs. The NO energy functional (0.36) reads

$$
E\left[N,\left\{n_{i}\right\},\left\{\phi_{i}(\mathbf{x})\right\}\right]=\sum_{i} n_{i} h_{i}^{i}+V_{e e}\left[N,\left\{n_{i}\right\},\left\{\phi_{i}(\mathbf{x})\right\}\right]
$$

We may conclude that the $1-\mathrm{RDM}$ and the functional $N$-representability problems are entirely different. The former is trivial solved since ONs sum up to the number of electrons $N$ and lie between 0 and 1 assure an $N$ - 
xiv

representable 1-RDM. The latter refers to the conditions that guarantee the one-to-one correspondence between $E\left[N,{ }^{2} \mathbf{D}\right]$ and $E\left[N,{ }^{1} \mathbf{D}\right]$, which is a related problem to the $N$-representability of the 2-RDM. Consequently, any approximation for $V_{e e}\left[N,\left\{n_{i}\right\},\left\{\phi_{i}(\mathbf{x})\right\}\right]$ must comply at least with the known necessary conditions for the $N$-representability of the 2-RDM.

\subsection{THE EULER EQUATIONS}

Minimization of the functional (0.41) has to be performed under the orthonormality requirement $(0.4)$ for the NSOs whereas the ONs conform to the $N$-representability conditions for ${ }^{1} \mathbf{D}$. Bounds on the ONs are enforced by setting $n_{i}=\cos ^{2} \gamma_{i}$ and varying $\gamma_{i}$ without constraints. The other two conditions may be easily taken into account by the method of Lagrange multipliers.

Associate the Lagrange multiplier $\mu$ (chemical potential) with the normalization condition (0.6), the set of Hermitian-Lagrange multipliers $\left\{\lambda_{k}^{i}\right\}$ with orthonormality constraints (0.4), and define the auxiliary functional $\Omega$ by the formula

$$
\Omega\left[N,\left\{\gamma_{i}\right\},\left\{\phi_{i}(\mathbf{x})\right\}\right]=E-\mu\left(\sum_{i} \cos ^{2} \gamma_{i}-N\right)-\sum_{i k} \lambda_{k}^{i}\left(\left\langle\phi_{k} \mid \phi_{i}\right\rangle-\delta_{i}^{k}\right)
$$

The functional (0.42) has to be stationary with respect to variations in $\left\{\phi_{i}(\mathbf{x})\right\},\left\{\phi_{i}^{*}(\mathbf{x})\right\}$ and $\left\{\gamma_{i}\right\}$

$$
\begin{gathered}
\delta \Omega=\sum_{i} \sin \left(2 \gamma_{i}\right)\left[\mu-\frac{\partial E}{\partial n_{i}}\right] d \gamma_{i}+\sum_{i} \int d \mathbf{x} \delta \phi_{i}^{*}(\mathbf{x})\left[\frac{\delta E}{\delta \phi_{i}^{*}(\mathbf{x})}-\sum_{k} \lambda_{i}^{k} \phi_{k}(\mathbf{x})\right] \\
+\sum_{i} \int d \mathbf{x}\left[\frac{\delta E}{\delta \phi_{i}(\mathbf{x})}-\sum_{k} \lambda_{k}^{i} \phi_{k}^{*}(\mathbf{x})\right] \delta \phi_{i}(\mathbf{x})=0
\end{gathered}
$$

The partial derivative $\left(\partial E / \partial n_{i}\right)$ is taken holding the orbitals fixed. It satisfies the relation

$$
\frac{\partial E}{\partial n_{i}}=h_{i}^{i}+\frac{\partial V_{e e}}{\partial n_{i}}=\mu
$$

For a fixed set of occupations, the orbital Euler equations are

$$
n_{i} \widehat{V}(\mathbf{x}) \phi_{i}(\mathbf{x})=\sum_{k} \lambda_{i}^{k} \phi_{k}(\mathbf{x})
$$

where

$$
\widehat{V}(\mathbf{x})=\widehat{h}(\mathbf{x})+\frac{1}{n_{i} \phi_{i}(\mathbf{x})} \frac{\delta V_{e e}}{\delta \phi_{i}^{*}(\mathbf{x})}
$$


Considering the orthonormality conditions (0.4), the elements of the Langrangian $\lambda$ read

$$
\lambda_{i}^{k}=V_{i}^{k} n_{i}=h_{i}^{k} n_{i}+\left(g_{k}^{i}\right)^{*} \quad\left(\lambda=\mathbf{h}^{1} \mathbf{D}+\mathbf{g}^{\dagger}\right)
$$

where the matrix $\mathbf{g}$ posses the elements

$$
g_{i}^{k}=\int d \mathbf{x} \frac{\delta V_{e e}}{\delta \phi_{k}(\mathbf{x})} \phi_{i}(\mathbf{x})
$$

A complex conjugated set of equations to (0.45) results for $\left\{\phi_{i}^{*}(\mathbf{x})\right\}$. Simultaneous solution of Eqs. (0.44)-(0.46) yields the optimum bound on the ground-state energy.

\subsubsection{The Gilbert nonlocal potential}

By making use of the Gilbert formal identity [15],

$$
\frac{\delta V_{e e}}{\delta \phi_{i}^{*}(\mathbf{x})}=n_{i} \phi_{i}(\mathbf{x}) \widehat{v}_{e e}(\mathbf{x})
$$

we may write the orbital Euler equations (0.45) in a convenient form:

$$
n_{i}\left[\widehat{h}(\mathbf{x})+\widehat{v}_{e e}(\mathbf{x})\right] \phi_{i}(\mathbf{x})=n_{i} \widehat{F}(\mathbf{x}) \phi_{i}(\mathbf{x})=\sum_{k} \lambda_{i}^{k} \phi_{k}(\mathbf{x})
$$

or in matrix representation

$$
F_{i}^{k} n_{i}=\lambda_{i}^{k}, \quad F_{i}^{k}=\lambda_{i}^{k} n_{i}^{-1} \quad\left(\mathbf{F}^{1} \mathbf{D}=\lambda, \quad \mathbf{F}=\lambda\left({ }^{1} \mathbf{D}\right)^{-1}\right)
$$

in which all matrices are Hermitean for a stationary state. Since the product of two Hermitian matrices is itself Hermitian if and only if they commute, it follows that the Hermiticity of $\lambda$ and Eq. (0.51) are together equivalent to the commutation relations

$$
\left[\mathbf{F},{ }^{1} \mathbf{D}\right]=0, \quad\left[\lambda,{ }^{1} \mathbf{D}\right]=0
$$

This implies that the 1-RDM and the Langrangian, $\lambda$, may be simultaneously brought to diagonal form by the same unitary transformation $\mathbf{U}$, from which follows

$$
\left[\widehat{h}(\mathbf{x})+\widehat{v}_{e e}(\mathbf{x})\right] \phi_{i}(\mathbf{x})=\lambda_{i} n_{i}^{-1} \phi_{i}(\mathbf{x})=\varepsilon_{i} \phi_{i}(\mathbf{x})
$$

The canonical orbitals thus satisfy concomitantly Eqs. (0.44) and (0.53), so we then have

$$
\left(v_{e e}\right)_{i}^{i}=\frac{\partial V_{e e}}{\partial n_{i}}, \quad \varepsilon_{i}=\frac{\lambda_{i}}{n_{i}}=\mu
$$


xvi

Assuming Eq. (0.49), this result shows that for canonical NSOs the operator $\widehat{F}$ has an essentially degenerate eigenvalue spectrum, i.e., all the NO eigenvalues are the same $(\mu)$, and are equal to minus the vertical IP [84]. Unfortunately, apart from the special case of the HF energy that may be viewed as the simplest 1-RDM functional, none of the currently known functionals (including the exact functional for the total energy in two-electron closed-shell systems) have effective potentials which satisfy the formal relation (0.49).

\subsubsection{Relation with the extended Koopman's theorem}

At present, the most known NOFs are defined in terms of NOs and ONs being only implicitly dependent on the 1-RDM. In this case, the energy functional still depends explicitly on the 2-RDM. As it was pointed out by Donnelly [18], there is a fundamental difference between energy functionals based explicitly on 1- and on 2-RDMs. The Eq. (0.45) for the optimum orbitals is actually the Löwdin's equation [82]. This equation cannot be reduced to an eigenvalue problem diagonalizing the matrix $\lambda$, although by slight manipulation the operator $\widehat{V}(\mathbf{x})$ can be transformed into a Hermitian operator with a nondegenerate spectrum of eigenvalues $\nu$. Such construction is provided by the extension of Koopmans' theorem [32, 33].

The equation for the EKT for ionization potentials may be derived by expressing the wavefunction of the $(N-1)$-electron system as a simple linear combination

$$
\left|\Psi^{N-1}\right\rangle=\sum_{i} C_{i} \widehat{a}_{i}\left|\Psi^{N}\right\rangle
$$

In Eq. (0.55), $\widehat{a}_{i}$ is the annihilation operator for an electron in orbital $i$, $\left|\Psi^{N}\right\rangle$ is the wavefunction of the $N$-electron system, $\left|\Psi^{N-1}\right\rangle$ is the wavefunction of the $(N-1)$-electron system and $\left\{C_{i}\right\}$ are a set of coefficients to be determined. Optimizing the energy of the state $\Psi^{N-1}$ with respect to the parameters $\left\{C_{i}\right\}$ and subtracting the energy of $\Psi^{N}$, gives the EKT equations as a generalized eigenvalue problem,

$$
\mathbf{T C}=\nu{ }^{1} \mathbf{D C}
$$

where $\nu$ are the EKT ionization potentials. In Eq. (0.56), the metric matrix is ${ }^{1} \mathbf{D}$ with the ONs $\left\{n_{i}\right\}$ along the diagonal and zeros in off-diagonal elements, and the transition matrix elements are given by

$$
T_{i}^{k}=\left\langle\Psi^{N}\left|\widehat{a}_{k}^{\dagger}\left[\widehat{H}, \widehat{a}_{i}\right]\right| \Psi^{N}\right\rangle=-\left(n_{i} h_{i}^{k}+2 \sum_{j l m}^{2} D_{l m}^{k j}\langle l m \mid i j\rangle\right)=-V_{i}^{k} n_{i}
$$


Eq. (0.56) can be transformed by canonical orthonormalization using $\left({ }^{1} \mathbf{D}\right)^{-1 / 2}$. With this transformation it can be written as

$$
\mathbf{T}^{\prime} \mathbf{C}^{\prime}=\nu \mathbf{C}^{\prime}
$$

It is now clear from Eqs. (0.47) and (0.57) that the diagonalization of the matrix $\nu$ with the elements

$$
\nu_{i}^{k}=-\frac{\lambda_{i}^{k}}{\sqrt{n_{k} n_{i}}}
$$

yields ionization potentials as eigenvalues [18, 72].

\subsubsection{The Pernal nonlocal potential}

Let's return to the problem of finding the optimal nonlocal potential $\widehat{v}_{e e}$ that the one-electron functions $\left\{\phi_{i}(\mathbf{x})\right\}$ resulting from solving the eigenproblem

$$
\widehat{F}(\mathbf{x}) \phi_{i}(\mathbf{x})=\left[\widehat{h}(\mathbf{x})+\widehat{v}_{e e}(\mathbf{x})\right] \phi_{i}(\mathbf{x})=\varepsilon_{i} \phi_{i}(\mathbf{x})
$$

satisfy the set of Euler equations (0.45).

Since the energy is real, the Langrangian $\lambda$ is a Hermitian matrix at the extremum,

$$
\lambda_{i}^{k}-\left(\lambda_{k}^{i}\right)^{*}=0 \quad\left(\lambda-\lambda^{\dagger}=0\right)
$$

From Eq. (0.61), by using Eq. (0.47), it follows without difficulty that

$$
\left(n_{i}-n_{k}\right) h_{i}^{k}+\left(g_{k}^{i}\right)^{*}-g_{i}^{k}=0 \quad\left(\left[\mathbf{h},{ }^{1} \mathbf{D}\right]+\mathbf{g}^{\dagger}-\mathbf{g}=0\right)
$$

This equation can be rewritten as a commutator between $\mathbf{F}$ and ${ }^{1} \mathbf{D}$,

$$
\left[\mathbf{F},{ }^{1} \mathbf{D}\right]=\left[\mathbf{h}+v_{e e},{ }^{1} \mathbf{D}\right]=0
$$

if we define the nonlocal potential matrix $v_{e e}$ by the commutation relation,

$$
\left(n_{i}-n_{k}\right)\left(v_{e e}\right)_{i}^{k}=\left(g_{k}^{i}\right)^{*}-g_{i}^{k} \quad\left(\left[v_{e e}{ }^{1} \mathbf{D}\right]=\mathbf{g}^{\dagger}-\mathbf{g}\right)
$$

According to Eq. (0.63), the 1-RDM and the generalized Fockian F commute at the extremum hence the NSOs are the solutions of the eigenproblem $(0.60)$ with the nonlocal potential defined by the identity (0.64). One should note however that Eq. (0.64) does not completely define $v_{e e}$. In fact, the diagonal elements $\left(v_{e e}\right)_{i}^{i}$ and the elements $\left(v_{e e}\right)_{i}^{k}$ corresponding to orbitals of equal ONs $\left(n_{i}=n_{k}\right)$ may be arbitrary. 
xviii

On the other hand, the Eq. (0.44) must be also satisfy by the optimal set of the NOs suggesting to define the diagonal elements of $\widehat{v}_{e e}$ by the formula

$$
\left(v_{e e}\right)_{i}^{i}=\frac{\partial V_{e e}}{\partial n_{i}}
$$

This selection implies that $\varepsilon_{i}=\mu$ for the optimal 1-RDM [compare with the Eq. (0.54)]. The Fockian matrix elements are as follows:

$$
F_{i}^{k}=h_{i}^{k}+\frac{\partial V_{e e}}{\partial n_{i}} \delta_{i}^{k}+\frac{1-\delta_{i}^{k}}{n_{i}-n_{k}} \int d \mathbf{x}\left[\phi_{k}^{*}(\mathbf{x}) \frac{\delta V_{e e}}{\delta \phi_{i}^{*}(\mathbf{x})}-\frac{\delta V_{e e}}{\delta \phi_{k}(\mathbf{x})} \phi_{i}(\mathbf{x})\right]
$$

In this manner, we have arrived at the Pernal nonlocal potential [81]. It can be shown, using the invariance of $V_{e e}$ with respect to an arbitrary unitary transformation and its extremal properties [13] or by means of the first-order perturbation theory applied to the eigenequation of the 1-RDM [81], that the off-diagonal elements of $\widehat{v}_{e e}$ may be also derived via the functional derivative

$$
\left(v_{e e}\right)_{i}^{k}=\frac{\partial V_{e e}}{\partial^{1} D_{k}^{i}}
$$

proposed by Gilbert [15].

The other degree of freedom in defining $\widehat{v}_{e e}$ related to the degeneracy of the 1-RDM can be avoided with a proper definition of the diagonal part $\left(v_{e e}\right)_{i}^{i}$ (see [81]). As a consequence, the NOs with the same ON are determined only up to the unitary transformation among them.

We conclude that the problem of finding optimal NOs turns into the iterative diagonalization of Eq. (0.60) with a Fockian matrix (0.66). The corresponding eigenfunctions are certainly orthonormal and optimize the total energy functional (0.41).

\subsection{CUMULANT OF THE 2-RDM}

The 2-RDM formulation (0.38) allows us to generalize the constrained search to approximately $N$-representable sets of 2 -RDMs. In order to approximate the unknown functional $V_{e e}\left[N,{ }^{1} \mathbf{D}\right]$, we use here a reconstructive functional ${ }^{2} \mathbf{D}\left[{ }^{1} \mathbf{D}\right]$, that is, we express the elements ${ }^{2} D_{k l}^{i j}$ in terms of the ${ }^{1} D_{k}^{i}$. We neglect any explicit dependence of ${ }^{2} \mathbf{D}$ on the NOs themselves because the energy functional has already a strong dependence on the NOs via the oneand two-electron integrals. 
The 2-RDM can be partitioned into an antisymmetrized product of the 1-RDMs, which is simply the HF approximation, and a correction ${ }^{2} \boldsymbol{\Gamma}$ to it,

$$
{ }^{2} D_{k l}^{i j}=\frac{1}{2}\left({ }^{1} D_{k}^{i}{ }^{1} D_{l}^{j}-{ }^{1} D_{k}^{j}{ }^{1} D_{l}^{i}\right)+{ }^{2} \Gamma_{k l}^{i j}=\frac{n_{j} n_{i}}{2}\left(\delta_{k}^{i} \delta_{l}^{j}-\delta_{k}^{j} \delta_{l}^{i}\right)+{ }^{2} \Gamma_{k l}^{i j}
$$

This decomposition of the 2-RDM is well-known from the cumulant theory (see chapter 4 ). ${ }^{2} \boldsymbol{\Gamma}$ is the cumulant matrix of the 2-RDM. Since it arises from interactions in the Hamiltonian, it might also be called the pair correlation matrix. This definition of correlation differs from the traditional one since ${ }^{1} \mathbf{D}$ is the one-matrix of the correlated system and not that corresponding to independent particles.

The first two terms on the rhs of Eq. (0.68) together satisfy properties (0.10)-(0.13) of the 2-RDM. Therefore, the cumulant matrix ${ }^{2} \boldsymbol{\Gamma}$ should satisfy these relations too. We further see that matrix elements of ${ }^{2} \boldsymbol{\Gamma}$ are nonvanishing only if all its labels refer to partially occupied NSOs with ON different from 0 or 1 . For a single Slater determinant the cumulant matrix vanishes.

It is important to note that the $2-\mathrm{RDM}$ is not additively separable (extensive), but its cumulant matrix ${ }^{2} \boldsymbol{\Gamma}$ satisfies this essential property. Finally, we must note that the trace of ${ }^{2} \boldsymbol{\Gamma}$ is of $O(N)$, i.e., it scales linearly with the size of the system, while the trace of the corresponding 2-RDM is of $O\left(N^{2}\right)$,

$$
\operatorname{Tr}\left({ }^{2} \boldsymbol{\Gamma}\right)=\sum_{i} \frac{n_{i}^{2}-n_{i}}{2}=O(N)
$$

It can be easily shown from Eqs. (0.24) and (0.25), taking into account the normalization condition (0.6) for each spin type, that spin components of ${ }^{2} \boldsymbol{\Gamma}$ fulfill the following sum rules:

$$
\begin{gathered}
2 \sum_{q}{ }^{2} \Gamma_{r \sigma, q \sigma}^{p \sigma, q \sigma}=n_{p}^{\sigma}\left(n_{p}^{\sigma}-1\right) \delta_{r}^{p} \\
\sum_{q}{ }^{2} \Gamma_{r \alpha, q \beta}^{p \alpha, q \beta}=0
\end{gathered}
$$

Using Eqs. (0.31), (0.32), (0.40) and (0.68) the energy Eq. (0.35) reads as

$$
\begin{aligned}
E & =\sum_{p}\left(n_{p}^{\alpha}+n_{p}^{\beta}\right) h_{p}^{p}+\frac{1}{2} \sum_{p q}\left(n_{q}^{\alpha}+n_{q}^{\beta}\right)\left(n_{p}^{\alpha}+n_{p}^{\beta}\right) J_{p q} \\
& -\frac{1}{2} \sum_{p q}\left(n_{q}^{\alpha} n_{p}^{\alpha}+n_{q}^{\beta} n_{p}^{\beta}\right) K_{p q}+\sum_{p q r t} 2 \widetilde{\Gamma}_{r t}^{p q}\langle r t \mid p q\rangle
\end{aligned}
$$

where $J_{p q}=\langle p q \mid p q\rangle$ and $K_{p q}=\langle p q \mid q p\rangle$ [see Eq. (0.3) with $\phi_{i}(\mathbf{x})$ replaced by $\left.\varphi_{p}(\mathbf{r})\right]$ are the usual Coulomb and exchange integrals, respectively. ${ }^{2} \widetilde{\Gamma}_{r t}^{p q}$ 
denotes the spinless cumulant matrix,

$$
{ }^{2} \widetilde{\Gamma}_{r t}^{p q}={ }^{2} \Gamma_{r \alpha, t \alpha}^{p \alpha, q \alpha}+{ }^{2} \Gamma_{r \alpha, t \beta}^{p \alpha, q \beta}+{ }^{2} \Gamma_{t \alpha, r \beta}^{q \alpha, p \beta}+{ }^{2} \Gamma_{r \beta, t \beta}^{p \beta, q \beta}
$$

Taking into account the sum rule (0.70), the expectation value of $\widehat{S}^{2}$ is likewise obtained from Eq. (0.29),

$$
\left\langle\widehat{S}^{2}\right\rangle=\frac{N^{\alpha}+N^{\beta}}{2}+\frac{\left(N^{\alpha}-N^{\beta}\right)^{2}}{4}-\sum_{p} n_{p}^{\alpha} n_{p}^{\beta}-\sum_{p q} 2{ }^{2} \Gamma_{q \alpha, p \beta}^{p \alpha, q \beta}
$$

\subsubsection{Approximate cumulant}

A large number of choices for the cumulant ${ }^{2} \boldsymbol{\Gamma}$ is possible. It has a dependence of four indices and direct computation with such magnitudes is too expensive to be applied to large systems. We want to maximize the physical content of ${ }^{2} \boldsymbol{\Gamma}$ to a few number of terms. We express ${ }^{2} \boldsymbol{\Gamma}$ by means of two-index matrices $\boldsymbol{\Delta}$ and $\boldsymbol{\Pi}$,

$$
\begin{aligned}
{ }^{2} \Gamma_{r \sigma_{1}^{\prime}, t \sigma_{2}^{\prime}}^{p \sigma_{1}, q \sigma_{2}} & =-\frac{\Delta_{q \sigma_{2}}^{p \sigma_{1}}}{2}\left(\delta_{r}^{p} \delta_{\sigma_{1}^{\prime}}^{\sigma_{1}} \delta_{t}^{q} \delta_{\sigma_{2}^{\prime}}^{\sigma_{2}}-\delta_{r}^{q} \delta_{\sigma_{1}^{\prime}}^{\sigma_{2}} \delta_{t}^{p} \delta_{\sigma_{2}^{\prime}}^{\sigma_{1}}\right) \\
& +\frac{m_{s}(1) m_{s}\left(1^{\prime}\right)}{2} \Pi_{t \sigma_{2}^{\prime}}^{p \sigma_{1}} \delta_{r}^{t} \delta_{-\sigma_{2}^{\prime}}^{\sigma_{1}^{\prime}} \delta_{p}^{q} \delta_{-\sigma_{2}}^{\sigma_{1}}
\end{aligned}
$$

Here $m_{s}$ denotes the sign of the spin projection (it takes two values +1 and -1). By taking into account the cumulant properties, Eqs. (0.10)-(0.13) with ${ }^{2} \mathbf{D}$ replaced by ${ }^{2} \boldsymbol{\Gamma}$, it can be shown that $\boldsymbol{\Delta}$ must be a real symmetric matrix $\left(\Delta_{q \sigma_{2}}^{p \sigma_{1}}=\Delta_{p \sigma 1}^{q \sigma_{2}}\right)$ with no unique diagonal elements whereas $\boldsymbol{\Pi}$ is a spin-independent $\left(\Pi_{r \alpha}^{p \alpha}=\Pi_{r \beta}^{p \alpha}=\Pi_{r \alpha}^{p \beta}=\Pi_{r \beta}^{p \beta}=\Pi_{r}^{p}\right)$ Hermitian matrix $\left[\Pi_{r}^{p}=\right.$ $\left.\left(\Pi_{p}^{r}\right)^{*}\right]$. We have the following spin structure for the cumulant matrix,

$$
\begin{aligned}
{ }^{2} \Gamma_{r \sigma, t \sigma}^{p \sigma, q \sigma} & =-\frac{\Delta_{q \sigma}^{p \sigma}}{2}\left(\delta_{r}^{p} \delta_{t}^{q}-\delta_{r}^{q} \delta_{t}^{p}\right) \\
{ }^{2} \Gamma_{r \alpha, t \beta}^{p \alpha, q \beta} & =-\frac{\Delta_{q \beta}^{p \alpha}}{2} \delta_{r}^{p} \delta_{t}^{q}+\frac{\Pi_{r}^{p}}{2} \delta_{r}^{t} \delta_{p}^{q}
\end{aligned}
$$

The sum rule (0.70) and the approximate ansatz (0.76) imply the constraint

$$
\sum_{q}{ }^{\prime} \Delta_{q \sigma}^{p \sigma}=n_{p}^{\sigma}\left(1-n_{p}^{\sigma}\right)
$$

where the prime indicates that the $q=p$ term is omitted. Analogously, one obtains using Eqs. (0.71) and (0.77) the following sum rule

$$
\sum_{q} \Delta_{q \beta}^{p \alpha}=\Pi_{p}^{p}
$$


By combining Eq. (0.72) with Eqs. (0.76) and (0.77), one arrives at the energy expression

$$
\begin{aligned}
E & =\sum_{p}\left(n_{p}^{\alpha}+n_{p}^{\beta}\right) h_{p}^{p}+\frac{1}{2} \sum_{p q}\left[\left(n_{q}^{\alpha}+n_{q}^{\beta}\right)\left(n_{p}^{\alpha}+n_{p}^{\beta}\right)-\widetilde{\Delta}_{q}^{p}\right] J_{p q} \\
& -\frac{1}{2} \sum_{p q}\left[\left(n_{q}^{\alpha} n_{p}^{\alpha}+n_{q}^{\beta} n_{p}^{\beta}\right)-\left(\Delta_{q \alpha}^{p \alpha}+\Delta_{q \beta}^{p \beta}\right)\right] K_{p q}+\sum_{p r} \Pi_{r}^{p} L_{p}^{r}
\end{aligned}
$$

where $\widetilde{\Delta}_{q}^{p}$ denotes the spinless $\boldsymbol{\Delta}$ matrix,

$$
\widetilde{\Delta}_{q}^{p}=\Delta_{q \alpha}^{p \alpha}+\Delta_{q \beta}^{p \alpha}+\Delta_{q \alpha}^{p \beta}+\Delta_{q \beta}^{p \beta}
$$

The new integral $L_{p}^{r}=\langle r r \mid p p\rangle$ arises from the correlation between particles with opposite spins and may be called the exchange and time-inversion integral [53]. In fact, one may obtain it as follows

$$
\begin{aligned}
& L_{p}^{r}=\int \frac{d \mathbf{x}_{1} d \mathbf{x}_{2}}{r_{12}}\left[\varphi_{r}^{\alpha}\left(\mathbf{r}_{1}\right) \alpha\left(\mathbf{s}_{1}\right)\right]^{*}\left[\varphi_{r}^{\beta}\left(\mathbf{r}_{2}\right) \beta\left(\mathbf{s}_{2}\right)\right]^{*} \varphi_{p}^{\alpha}\left(\mathbf{r}_{1}\right) \alpha\left(\mathbf{s}_{1}\right) \varphi_{p}^{\beta}\left(\mathbf{r}_{2}\right) \beta\left(\mathbf{s}_{2}\right)= \\
& \int \frac{d \mathbf{x}_{1} d \mathbf{x}_{2}}{r_{12}}\left[\varphi_{r}^{\alpha}\left(\mathbf{r}_{1}\right) \alpha\left(\mathbf{s}_{1}\right)\right]^{*}\left[\widehat{I} \varphi_{p}^{\alpha}\left(\mathbf{r}_{2}\right) \alpha\left(\mathbf{s}_{2}\right)\right]^{*} \widehat{I}(2) \widehat{P}_{12} \varphi_{r}^{\alpha}\left(\mathbf{r}_{1}\right) \alpha\left(\mathbf{s}_{1}\right) \varphi_{p}^{\alpha}\left(\mathbf{r}_{2}\right) \alpha\left(\mathbf{s}_{2}\right)
\end{aligned}
$$

or, equivalently,

$$
L_{p}^{r}=\langle r r \mid p p\rangle=\left\langle r \alpha, r \beta\left|r_{12}^{-1}\right| p \alpha, p \beta\right\rangle=\left\langle r \alpha, p \alpha\left|\widehat{I}^{\dagger}(2) r_{12}^{-1} \widehat{I}(2) \widehat{P}_{12}\right| r \alpha, p \alpha\right\rangle
$$

where $\widehat{P}_{12}$ operator permutes electrons 1 and 2 , and the time-inversion antiunitary operator $\widehat{I}$ changes a ket vector into bra vector and $\alpha(\beta)$ into $\beta(\alpha)$, i.e.,

$$
\widehat{I}|p \alpha\rangle=\langle p \beta|, \quad \widehat{I}| p \beta\rangle=-\langle p \alpha|
$$

Note that if $\Delta_{q \sigma^{\prime}}^{p \sigma}=0$ and $\Pi_{r}^{p}=0$, then the reconstruction proposed here yields the HF case as expected.

Similarly, combining Eq. (0.74) with Eqs. (0.76), (0.77) and (0.78) one arrives at the average total spin

$$
\begin{aligned}
\left\langle\widehat{S}^{2}\right\rangle & =\frac{N^{\alpha}+N^{\beta}}{2}+\frac{\left(N^{\alpha}-N^{\beta}\right)^{2}}{4} \\
& +\sum_{p}\left(\Delta_{p \beta}^{p \alpha}-n_{p}^{\alpha} n_{p}^{\beta}\right)-\sum_{p} \Pi_{p \beta}^{p \alpha}
\end{aligned}
$$

In Eq. (0.84), the $\alpha$ - and $\beta$-dependences of $\Pi_{p \beta}^{p \alpha}$ have been retained only to emphasize that it is related to these SOs. We recall that $\Pi$ is a spinindependent matrix as a consequence of the antisymmetric properties of cumulant ${ }^{2} \boldsymbol{\Gamma}$. 
xxii

\subsection{RESTRICTED CLOSED-SHELL NOF}

So far in this chapter we have discussed the NOF theory in terms of general set of SOs $\left\{\phi_{i}(\mathbf{x})\right\}$ or in terms of restricted SOs, which are constrained to have the same spatial function $\left\{\varphi_{p}(\mathbf{r})\right\}$ for $\alpha$ and $\beta$ spin functions. In this section we are concerned only with closed-shell systems. Our molecules are thus allowed to have only an even number of electrons, with all electrons paired such that the spatial orbitals are doubly occupied. In this case of spin compensated systems, the two non-zero blocks of the 1-RDM are the same $\left({ }^{1} \mathbf{D}_{\alpha}^{\alpha}={ }^{1} \mathbf{D}_{\beta}^{\beta}\right)$, i.e.,

$$
n_{p}^{\alpha}=n_{p}^{\beta}=n_{p}
$$

The trace of the one-matrix (0.6) becomes

$$
\sum_{p} n_{p}=N^{\alpha}=N^{\beta}=\frac{N}{2}
$$

and from Eq. (0.20) follows that

$$
\left\langle\widehat{S}_{z}\right\rangle=\frac{N^{\alpha}-N^{\beta}}{2}=0
$$

For singlet states, the first and the last blocks of the 2-RDM are also equal $\left({ }^{2} \mathbf{D}_{\alpha \alpha}^{\alpha \alpha}={ }^{2} \mathbf{D}_{\beta \beta}^{\beta \beta}\right)$, so hereafter we deal only with ${ }^{2} \mathbf{D}_{\alpha \alpha}^{\alpha \alpha}$ and ${ }^{2} \mathbf{D}_{\alpha \beta}^{\alpha \beta}$. We assume further that $\Delta_{q \alpha}^{p \alpha}=\Delta_{q \beta}^{p \alpha}=\Delta_{q \alpha}^{p \beta}=\Delta_{q \beta}^{p \beta}=\Delta_{q}^{p}$. For convenient purposes as we see below, we define the matrix $\boldsymbol{\Pi}$ in terms of a new spin-independent Hermitian matrix $\boldsymbol{\Lambda}$ :

$$
\Pi_{r}^{p}=n_{r} n_{p}-\Delta_{r}^{p}-\Lambda_{r}^{p}
$$

Combining both sum rules (0.78) and (0.79) with Eq. (0.88) results in

$$
\begin{aligned}
\sum_{q}{ }^{\prime} \Delta_{q}^{p} & =n_{p}\left(1-n_{p}\right) \\
2 \Delta_{p}^{p}+\Lambda_{p}^{p} & =2 n_{p}^{2}-n_{p}
\end{aligned}
$$

Taking into account Eq. (0.80), the closed-shell energy can be expressed as

$$
E=2 \sum_{p} n_{p} h_{p}^{p}+\sum_{p q}\left(n_{q} n_{p}-\Delta_{q}^{p}\right)\left(2 J_{p q}-K_{p q}+L_{p}^{q}\right)-\sum_{p r} \Lambda_{r}^{p} L_{p}^{r}
$$




\subsubsection{Two-electron systems}

NOF theory provides an exact energy functional for two-electron systems $[76,77]$. In the weak correlation limit, the total energy is given by

$$
E=2 \sum_{p=1}^{\infty} n_{p} h_{p}^{p}+n_{1} L_{1}^{1}-2 \sum_{p=2}^{\infty} \sqrt{n_{1} n_{p}} L_{p}^{1}+\sum_{p, r=2}^{\infty} \sqrt{n_{r} n_{p}} L_{p}^{r}
$$

As can be seen from Eq. (0.92), the dependence of the 2-RDM on the ONs requires a distinction between spatial orbitals.

Since ${ }^{2} \mathbf{D}_{\alpha \alpha}^{\alpha \alpha}=0$ for $N=2$, one easily deduces from Eqs. (0.68) and (0.76) that $\Delta_{q}^{p}=n_{q} n_{p}$. Consequently, it is not difficult to see from Eqs. (0.68), (0.77) and (0.88) that ${ }^{2} \mathbf{D}_{\alpha \beta}^{\alpha \beta}$ nonzero elements have the form ${ }^{2} D_{r \alpha, r \beta}^{p \alpha, p \beta}=-\Lambda_{r}^{p} / 2$. Thus, the total energy (0.91) turns into

$$
E=2 \sum_{p} n_{p} h_{p}^{p}-\sum_{p r} \Lambda_{r}^{p} L_{p}^{r}
$$

From the requirement that for any two-electron system the expression (0.93) should yield Eq. (0.92), one has to set

$$
\Lambda_{r}^{p}=-n_{p} \delta_{r}^{p}+\left(1-\delta_{r}^{p}\right)\left[1-2 \theta\left(0.5-n_{r}\right) \theta\left(0.5-n_{p}\right)\right] \sqrt{n_{r} n_{p}}
$$

where $\theta(x)$ is the unit step function also known as the Heaviside function.

It is worth noting that the chosen $\boldsymbol{\Delta}$ and $\boldsymbol{\Lambda}$ satisfy the constraints $(0.78)$ and (0.90). Moreover, the expression (0.94) also helds true for systems where the largest occupation deviates significantly from one, indicating that it may be possibly valid for arbitrary correlation strengths [76].

\subsubsection{N-electron systems}

Let us assume the functional form (0.94) for the matrix $\boldsymbol{\Lambda}$ in the general case of $N$-electron systems. This assumption and the equality (0.90), affords

$$
\Lambda_{p}^{p}=-n_{p}, \quad \Delta_{p}^{p}=n_{p}^{2}
$$

or, equivalently,

$$
\Lambda_{p \sigma^{\prime}}^{p \sigma}=-\sqrt{n_{p}^{\sigma} n_{p}^{\sigma^{\prime}}}=-n_{p}, \quad \Delta_{p \sigma^{\prime}}^{p \sigma}=n_{p}^{\sigma} n_{p}^{\sigma^{\prime}}=n_{p}^{2}
$$

By taking into account Eq. (0.96), last two terms in Eq. (0.84) are found

$$
\sum_{p}\left(\Delta_{p \beta}^{p \alpha}-n_{p}^{\alpha} n_{p}^{\beta}\right)=0, \quad \sum_{p} \Pi_{p \beta}^{p \alpha}=\frac{N}{2}
$$

which leads to the exact result $<\widehat{S}^{2}>=0$ for singlet states $(2 S+1=1)$. 
xxiv

Very different functional form is expected for $\boldsymbol{\Delta}$ in molecules with more than two electrons. Without any calculations it is clear that $\mathrm{H}_{2}$ is atypical, since ${ }^{2} \mathbf{D}_{\alpha \alpha}^{\alpha \alpha}$ vanishes for this system. $\Delta_{q}^{p}=n_{q} n_{p}(q \neq p)$, taken from the $N=2$ case, violates the sum rule (0.89) in the general case of $N>2$. This means that the functional form of nondiagonal elements of $\boldsymbol{\Delta}$ is unknown for $N$-electron systems, as yet. Nevertheless, some constraints can be achieved for these quantities using known necessary conditions of 2-RDM $N$-representability.

The mentioned above positivity conditions state that the $2-\mathrm{RDM}^{2} \mathbf{D}$, the electron-hole density matrix $\mathbf{G}$, and the two-hole density matrix $\mathbf{Q}$ must be positive semidefinite. A matrix is positive semidefinite if and only if all of its eigenvalues are nonnegative. The solution of the corresponding eigenproblems is readily carried out [73]. For ${ }^{2} \mathbf{D}$, it yields the following set of eigenvalues:

$$
d=\left\{0, n_{q} n_{p}-\Delta_{q}^{p}, \frac{1}{2}\left(n_{q} n_{p}-\Delta_{q}^{p}\right)\right\}, \quad q \neq p
$$

There is also a single $R \times R$ block of the spin component ${ }^{2} \mathbf{D}_{\alpha \beta}^{\alpha \beta}$, which has elements equal to $\Pi_{r}^{p} / 2$. Accordingly, we have analytic expressions for all eigenvalues of ${ }^{2} \mathbf{D}$, except those arising from the single $R \times R$ block. Consequently, our reconstructive functional satisfies the D-condition $(d \geq 0)$ if $\Delta_{q}^{p} \leq n_{q} n_{p}$ and the $R \times R$ block is positive.

Considering that $\mathbf{Q}$ has the same block structure as ${ }^{2} \mathbf{D}$, one obtains analogously that if one takes $\Delta_{q}^{p} \leq\left(1-n_{q}\right)\left(1-n_{p}\right)$, and the $R \times R$ block of $\mathbf{Q}_{\alpha \beta}^{\alpha \beta}$ is positive, the Q-condition is fulfilled. Consequently, D-condition is more restrictive than Q-condition between orbitals with ONs close to zero, whereas for $\boldsymbol{\Delta}$-elements between orbitals with ONs close to one, the Q-condition is predominant.

The spin component $\mathbf{G}_{\alpha \alpha}^{\alpha \alpha}$ contains a single block $R \times R$, for which the eigenvalues have no analytic expression, and $1 \times 1$ blocks. The latter blocks have nonnegative eigenvalues if $\Delta_{q}^{p} \geq n_{p}\left(n_{q}-1\right)$. This inequality is easy to satisfy on the domain of allowed ONs $\left(n_{q} \leq 1\right)$ if we consider nonnegative $\Delta_{q}^{p}$.

The opposite spin component consists entirely of $1 \times 1$ blocks $G_{p \alpha, p \beta}^{p \alpha, p \beta}=0$, and $2 \times 2$ blocks which afford the eigenvalues

$$
g_{q}^{p}=\frac{n_{q}+n_{p}}{4}+\frac{\Delta_{q}^{p}-n_{q} n_{p}}{2} \pm \frac{1}{4} \sqrt{\left(n_{q}-n_{p}\right)^{2}+4\left(n_{q} n_{p}-\Delta_{q}^{p}-\Lambda_{q}^{p}\right)^{2}}
$$

In order to ensure that $g_{q}^{p} \geq 0$, the expression (0.99) gives rise to the inequality

$$
\Delta_{q}^{p} \leq n_{q} n_{p}+\frac{n_{q} n_{p}-\left(\Lambda_{q}^{p}\right)^{2}}{2 \Lambda_{q}^{p}-n_{q}-n_{p}}=n_{q} n_{p}
$$

Concluding, to satisfy the known necessary $N$-representability conditions for the 2-RDM, the matrix elements of $\boldsymbol{\Delta}$ has to conform the following analytic 
constraints:

$$
\Delta_{q}^{p} \leq n_{q} n_{p}, \quad \Delta_{q}^{p} \leq\left(1-n_{q}\right)\left(1-n_{p}\right), \Delta_{q}^{p} \geq n_{p}\left(n_{q}-1\right), \quad q \neq p
$$

\subsubsection{Effective one-electron operator}

The best NSOs are those which minimize the electronic energy subject to orthonormality constraints (0.4), and hence satisfy Löwdin's Eqs. (0.45)(0.46). For the energy functional (0.91), these equations become into the spatial orbital Euler equations

$$
n_{p} \widehat{V}^{p}\left(\mathbf{r}_{1}\right) \varphi_{p}\left(\mathbf{r}_{1}\right)=\sum_{r} \lambda_{p}^{r} \varphi_{r}\left(\mathbf{r}_{1}\right)
$$

where

$$
\begin{aligned}
\widehat{V}^{p}\left(\mathbf{r}_{1}\right) & =\widehat{h}\left(\mathbf{r}_{1}\right)+\widehat{v}^{p}\left(\mathbf{r}_{1}\right) \\
\widehat{v}^{p}\left(\mathbf{r}_{1}\right) & =\sum_{q}{ }^{\prime}\left(n_{q}-\frac{\Delta_{q}^{p}}{n_{p}}\right)\left[2 \widehat{J}_{q}\left(\mathbf{r}_{1}\right)-\widehat{K}_{q}\left(\mathbf{r}_{1}\right)+\widehat{L}_{q}\left(\mathbf{r}_{1}\right)\right] \\
& -\sum_{q} \frac{\Lambda_{q}^{p}}{n_{p}} \widehat{L}_{q}\left(\mathbf{r}_{1}\right) \\
\widehat{J}_{q}\left(\mathbf{r}_{1}\right) & =\int d \mathbf{r}_{2} \varphi_{q}^{*}\left(\mathbf{r}_{2}\right) r_{12}^{-1} \varphi_{q}\left(\mathbf{r}_{2}\right) \\
\widehat{K}_{q}\left(\mathbf{r}_{1}\right) & =\int d \mathbf{r}_{2} \varphi_{q}^{*}\left(\mathbf{r}_{2}\right) r_{12}^{-1} \widehat{P}_{12} \varphi_{q}\left(\mathbf{r}_{2}\right) \\
\widehat{L}_{q}\left(\mathbf{r}_{1}\right) & =\int d \mathbf{r}_{2} \varphi_{q}\left(\mathbf{r}_{2}\right) r_{12}^{-1} \widehat{I}(2) \widehat{P}_{12} \varphi_{q}\left(\mathbf{r}_{2}\right)
\end{aligned}
$$

where $\widehat{J}_{q}\left(\mathbf{r}_{1}\right)$ and $\widehat{K}_{q}\left(\mathbf{r}_{1}\right)$ are the usual Coulomb and exchange operators, respectively, whereas $\widehat{L}_{q}\left(\mathbf{r}_{1}\right)$ is the exchange and time-inversion operator [53]. Here, $\widehat{I}(2)$ becomes just into the complex conjugate operator. It is important to note that the operator $\widehat{V}^{p}\left(\mathbf{r}_{1}\right)$ does not fulfill the Gilbert relation (0.49). Consequently, Eq. (0.102) cannot be reduced to an eigenvalue problem diagonalizing the matrix $\lambda$.

On the other hand, the one-electron functions $\left\{\varphi_{p}(\mathbf{r})\right\}$ that satisfy the set of Eq. (0.102) may be obtained from solving the eigenproblem (0.60) with the Fockian matrix [see Eq. (0.66)]

$$
F_{p}^{r}=h_{p}^{r}+\left(v_{e e}\right)_{p}^{r}
$$


xxvi

where the nonlocal potential matrix elements are

$$
\begin{aligned}
\left(v_{e e}\right)_{p}^{r} & =\frac{\partial V_{e e}}{\partial n_{p}} \delta_{p}^{r}+\frac{1-\delta_{p}^{r}}{n_{p}-n_{r}} \int d \mathbf{r} \varphi_{r}^{*}(\mathbf{r})\left[n_{p} \widehat{v}^{p}(\mathbf{r})-n_{r} \widehat{v}^{r}(\mathbf{r})\right] \varphi_{p}(\mathbf{r}) \\
\frac{\partial V_{e e}}{\partial n_{p}} & =L_{p}^{p}+\sum_{q}^{\prime}\left(n_{q}-\frac{\partial \Delta_{q}^{p}}{\partial n_{p}}\right)\left(2 J_{p q}-K_{p q}\right) \\
& +\sum_{q}^{\prime}\left(n_{q}-\frac{\partial \Delta_{q}^{p}}{\partial n_{p}}-\frac{\partial \Lambda_{q}^{p}}{\partial n_{p}}\right)\left(\frac{L_{p}^{q}+L_{q}^{p}}{2}\right)
\end{aligned}
$$

Let us assume that the set of spatial orbitals $\left\{\varphi_{p}(\mathbf{r})\right\}$ is real, then $L_{p}^{q}=$ $L_{q}^{p}=K_{p q}$, which allows Eqs. (0.104) and (0.110) to be further simplified, yielding

$$
\begin{aligned}
\widehat{v}^{p}(\mathbf{r}) & =\widehat{J}_{p}(\mathbf{r})+2 \sum_{q}{ }^{\prime}\left(n_{q}-\frac{\Delta_{q}^{p}}{n_{p}}\right) \widehat{J}_{q}(\mathbf{r})-\sum_{q}{ }^{\prime} \Lambda_{q}^{p} \widehat{K}_{q}(\mathbf{r}) \\
\frac{\partial V_{e e}}{\partial n_{p}} & =J_{p p}+2 \sum_{q}^{\prime}\left(n_{q}-\frac{\partial \Delta_{q}^{p}}{\partial n_{p}}\right) J_{p q}-\sum_{q}{ }^{\prime} \frac{\partial \Lambda_{q}^{p}}{\partial n_{p}} K_{p q}
\end{aligned}
$$

The closed-shell energy (0.91) for real orbitals can be rewritten as

$$
E=\sum_{p}\left(2 h_{p}^{p}+J_{p p}\right) n_{p}+2 \sum_{p q}{ }^{\prime}\left(n_{q} n_{p}-\Delta_{q}^{p}\right) J_{p q}-\sum_{p q}{ }^{\prime} \Lambda_{q}^{p} K_{p q}
$$

\subsection{RESTRICTED OPEN-SHELL NOF}

We consider now situations in which a molecule has one or more unpaired electrons, hence we may have spin-polarized systems. The spatial orbitals are thus divided into two categories. Namely, those which are double occupied with two electrons of opposite spin $\left(n_{p}^{\alpha}\right.$ and $\left.n_{p}^{\beta}\right)$, called closed shells $(c l)$, and singly occupied $\left(n_{i}^{\alpha}\right.$ or $\left.n_{i}^{\beta}\right)$, called open shells $(o p)$. We assume further spinindependent ONs. All electron spins corresponding to the closed shell part are then paired $\left(n_{p}^{\alpha}=n_{p}^{\beta}=n_{p}\right)$ and thus they are coupled as a pure singlet. By the help of the following result [last term in Eq. (0.84)]

$$
\sum_{p} \Pi_{p \beta}^{p \alpha}=\sum_{p} \sqrt{n_{p}^{\alpha} n_{p}^{\beta}}=\sum_{p}^{c l} n_{p}=\frac{N_{c l}}{2}
$$


it is easily to calculate [see Eqs. (0.20) and (0.84)] the expectation values for spin operators $\widehat{S}_{z}$ and $\widehat{S}^{2}$, in particular,

$$
\begin{aligned}
\left\langle\widehat{S}_{z}\right\rangle & =\sum_{p}^{o p} \frac{n_{p}^{\alpha}-n_{p}^{\beta}}{2}=\frac{N_{o p}^{\alpha}-N_{o p}^{\beta}}{2} \\
\left\langle\widehat{S}^{2}\right\rangle & =\frac{N_{o p}^{\alpha}}{2}\left(\frac{N_{o p}^{\alpha}}{2}+1\right)+\frac{N_{o p}^{\beta}}{2}\left(\frac{N_{o p}^{\beta}}{2}+1\right)-\frac{1}{2} N_{o p}^{\alpha} N_{o p}^{\beta}
\end{aligned}
$$

The situation here is completely analogous to that obtained in the restricted open HF theory (ROHF). The states are not eigenfunctions of $\widehat{S}^{2}$, except when all the open-shell electrons have parallel spins $\left(N_{o p}^{\alpha}=0\right.$ or $\left.N_{o p}^{\beta}=0\right)$. This result is a consequence of the expansion (0.22) used to obtain Eq. (0.27). Actually, the spin decomposition $(0.22)$ for ${ }^{2} \mathbf{D}$ does not conserve in general the total spin $S$. However, we can form appropriate linear combinations of two-electron spin functions $\left\{\sigma_{n}^{(2)}\left(\mathbf{s}_{1}^{\prime}, \mathbf{s}_{2}^{\prime} \mid \mathbf{s}_{1}, \mathbf{s}_{2}\right)\right\}$ which are simultaneously eigenfunctions of $\widehat{S}_{z}$ and $\widehat{S}^{2}$, and achieve a correct spin decomposition of the 2-RDM [85],

$$
{ }^{2} D\left(\mathbf{x}_{1}^{\prime}, \mathbf{x}_{2}^{\prime} \mid \mathbf{x}_{1}, \mathbf{x}_{2}\right)=\sum_{n=1}^{6}{ }^{2} \mathbf{D}^{n}\left(\mathbf{r}_{1}^{\prime}, \mathbf{r}_{2}^{\prime} \mid \mathbf{r}_{1}, \mathbf{r}_{2}\right) \sigma_{n}^{(2)}\left(\mathbf{s}_{1}^{\prime}, \mathbf{s}_{2}^{\prime} \mid \mathbf{s}_{1}, \mathbf{s}_{2}\right)
$$

where, for example,

$$
\sigma_{1}^{(2)}\left(\mathbf{s}_{1}^{\prime}, \mathbf{s}_{2}^{\prime} \mid \mathbf{s}_{1}, \mathbf{s}_{2}\right)=\frac{1}{2}\left[\alpha^{*} \alpha^{*} \alpha \alpha+\beta^{*} \beta^{*} \beta \beta+\alpha^{*} \beta^{*} \alpha \beta+\beta^{*} \alpha^{*} \beta \alpha\right]
$$

Afterward, we have to find approximations for 2-RDM spin-components $\left\{{ }^{2} \mathbf{D}^{n}\right\}$.

Let us now focus on high-spin cases only, such as doublet, triplet, quartet ... spins for one, two, three ... unpaired electrons outside the closed shells. Accordingly, singly occupied orbitals will always have the same spin $\left(N_{o p}^{\alpha}=0\right.$ or $\left.N_{o p}^{\beta}=0\right)$ so the trace of the one-matrix (0.6) becomes

$$
2 \sum_{p}^{c l} n_{p}+\sum_{p}^{o p} n_{p}=N_{c l}+N_{o p}=N
$$


xxviii

In fact, the value of $N_{o p}$ is determined by the conservation of the spin, so we have two constraints

$$
\begin{aligned}
& \frac{1}{2} \sum_{p}^{o p} n_{p}=\frac{1}{2} N_{o p}=S \\
& 2 \sum_{p}^{c l} n_{p}=N_{c l}=N-2 S
\end{aligned}
$$

where $S$ is the quantum number describing the total spin of the $N$-electron high-spin coupled multiplet state.

We assume further that the ON of the open shell $p$ is always one $\left(n_{p}=1\right)$. This assumption is trivial for a doublet, but it is more restrictive for higher multiplet with a corresponding underestimation of the energy. It should be reminded that matrix elements of ${ }^{2} \boldsymbol{\Gamma}$ are nonvanishing only if all its labels refer to partially occupied NOs therefore $\boldsymbol{\Delta}=0$ and $\boldsymbol{\Pi}=0$ if we consider a cumulant made up of at least one open-shell level. Since $\boldsymbol{\Delta}$ and $\boldsymbol{\Pi}$ refer only to closed shells, we consider them spin-independent. The sum rule (0.89) becomes

$$
\sum_{q}^{c l}{ }^{\prime} \Delta_{q}^{p}=n_{p}\left(1-n_{p}\right)
$$

and the energy expression (0.80) for such a system is

$$
\begin{aligned}
E & =\sum_{p}^{c l}\left(2 h_{p}^{p}+J_{p p}\right) n_{p}+2 \sum_{p q}^{c l}{ }^{\prime}\left(n_{q} n_{p}-\Delta_{q}^{p}\right) J_{p q}-\sum_{p q}^{c l}{ }^{\prime} \Lambda_{q}^{p} K_{p q} \\
& +\sum_{p}^{c l} \sum_{q}^{o p} n_{p}\left(2 J_{p q}-K_{p q}\right)+\sum_{p}^{o p} h_{p p}+\frac{1}{2} \sum_{p q}^{o p}\left(J_{p q}-K_{p q}\right)
\end{aligned}
$$

where we have considered real orbitals $\left(L_{q}^{p}=K_{p q}\right)$.

\subsection{PRACTICAL NOF}

Electronic structure computations would be greatly simplified by the finding of practical NOFs. One may attempt to approximate the unknown off-diagonal elements of $\boldsymbol{\Delta}$ considering the sum rule (0.89) and analytic constraints (0.101) imposed by the D-, G- and Q-conditions. However, it is not evident how to approach $\Delta_{q}^{p}$, for $p \neq q$, in terms of the ONs. Due to this fact, let's rewrite the energy term which involves $\boldsymbol{\Delta}$ as

$$
\sum_{p q}{ }^{\prime} \Delta_{q}^{p} J_{p q}=\sum_{p} J_{p}^{*} \sum_{q}{ }^{\prime} \Delta_{q}^{p}
$$


where $J_{p}^{*}$ denotes the mean value of the Coulomb interactions $J_{p q}$ for a given orbital $p$ taking over all orbitals $q \neq p$. From the property shown in Eq. (0.89) follows immediately

$$
\sum_{p q}{ }^{\prime} \Delta_{q}^{p} J_{p q}=\sum_{p} n_{p}\left(1-n_{p}\right) J_{p}^{*}
$$

Inserting this expression into Eq. (0.114), one obtains

$$
\begin{aligned}
E^{c l} & =\sum_{p}\left(2 n_{p} h_{p}^{p}+n_{p}^{2} J_{p p}\right)+\sum_{p q}{ }^{\prime}\left(2 n_{q} n_{p} J_{p q}-\Lambda_{q}^{p} K_{p q}\right) \\
& +\sum_{p} n_{p}\left(1-n_{p}\right)\left(J_{p p}-2 J_{p}^{*}\right)
\end{aligned}
$$

A further simplification is accomplished by setting $J_{p}^{*} \approx J_{p p} / 2$, which produces

$$
E^{c l}=\sum_{p}\left(2 n_{p} h_{p}^{p}+n_{p}^{2} J_{p p}\right)+\sum_{p q}^{\prime}\left(2 n_{q} n_{p} J_{p q}-\Lambda_{q}^{p} K_{p q}\right)
$$

The high-spin open-shell energy expression can be obtained in completely similar manner from Eq. (0.124),

$$
\begin{aligned}
E^{o p} & =\sum_{p}^{c l}\left(2 n_{p} h_{p}^{p}+n_{p}^{2} J_{p p}\right)+\sum_{p q}^{c l}{ }^{\prime}\left(2 n_{q} n_{p} J_{p q}-\Lambda_{q}^{p} K_{p q}\right) \\
& +\sum_{p}^{c l} \sum_{q}^{o p} n_{p}\left(2 J_{p q}-K_{p q}\right)+\sum_{p}^{o p} h_{p p}+\frac{1}{2} \sum_{p q}^{o p}\left(J_{p q}-K_{p q}\right)
\end{aligned}
$$

We have thus arrived at an approximate NOF which coincides with the selfinteraction-corrected Hartree functional proposed by Goedecker and Umrigar except for the choice of phases given by the sign of $\left(\Lambda_{q}^{p}\right)$. Unfortunately, this NOF gives a wrong description of the ONs for the lowest occupied orbitals. In order to ensure that these ONs only are close to unity we propose to add a new term to the functional form (0.94) of matrix $\boldsymbol{\Lambda}$, namely,

$$
\begin{aligned}
\Lambda_{r}^{p} & =-n_{p} \delta_{r}^{p}+\left(1-\delta_{r}^{p}\right)\left[1-2 \theta\left(0.5-n_{r}\right) \theta\left(0.5-n_{p}\right)\right] \sqrt{n_{r} n_{p}} \\
& +\left(1-\delta_{r}^{p}\right) \theta\left(n_{r}-0.5\right) \theta\left(n_{p}-0.5\right) \sqrt{\left(1-n_{r}\right)\left(1-n_{p}\right)}
\end{aligned}
$$

\subsection{NUMERICAL IMPLEMENTATION}

In section 4 , we have obtained the Euler equations (0.44)-(0.46) which yield the optimum bound on the ground-state energy. Usually, the simultaneous 
solution of these equations is established with an embedded loop algorithm. In the inner loop we look for the optimal ONs for a given set of orbitals fulfilling Eq. (0.44), whereas in the outer loop we optimize the NOs under the orthonormality condition for fixed ONs. We recall that bounds on the ONs are enforced by setting $n_{i}=\cos ^{2} \gamma_{i}$.

An important task in NOF theory is to find an efficient procedure for carrying out the orbital optimization. Direct minimization has been proven $[44,47,76]$ to be a costly method. First, there are directions at the energy minima with very low curvature associated with the high-energy NOs. The latter have very small ONs and hence give very small contribution to the energy. There is also a need for other starting orbitals that are closer to the optimized ones than are the HF orbitals, which are poor initial guesses in this iterative procedure, and lead to lengthy orbital optimizations. Finally, and most critically, is that the NO coefficient matrix must be reorthogonalized during the course of the optimization which is the rate-limiting step.

The one-electron equations (0.60) offer a new possibility for finding the optimal NSOs by iterative diagonalization of the Fockian (0.66). The main advantage of this method is that the resulting orbitals are automatically orthogonal. The first calculations based on this diagonalization technique has confirmed its practical value [81].

\subsection{RESULTS}

The study of different properties provides a measure of accuracy that can be employed in testing approximate functionals. In this section, we just quote some relevant results for selected molecules. Both the inner- and outer-loop optimizations were implemented using a sequential quadratic programming (SQP) method [86] which, computationally speaking, is a very demanding algorithm. Accordingly, we have chosen Pople medium-size basis sets [87] for the calculations and we have compared our results with the results using

other methods at the same level. Among the approaches compared are the coupled cluster technique including all single and double excitations and a perturbational estimate of the connected triple excitations $[\mathrm{CCSD}(\mathrm{T})]$, as well as the Becke-3-Lee-Yang-Parr (B3LYP) density functional [88]. The CC and B3LYP values were calculated with the GAUSSIAN system of programs [89], using the basis set keyword 5D.

\subsubsection{Energetics}

In Table I and II we report the values obtained for the total energies employing the experimental geometry [90].

\section{[ TABLE I ]}


RESULTS XXxi

A survey of these Tables reveals that NOF values are more like CCSD $(T)$ calculations, which are very accurate results for the basis-set correlation energies on these small molecules. The B3LYP values, as is well known, tend to be too low. We note that the percentage of the correlation energy obtained by $\operatorname{CCSD}(\mathrm{T})$ decreases as the number of electrons increases, whereas our functional keeps giving a slightly larger portion of the correlation energy (e.g. $\mathrm{P}_{2}$, $\left.\mathrm{SO}_{2}, \mathrm{Cl}_{2}\right)$.

\section{[ TABLE II ]}

The EKT provides an alternative assessment for approximate NOFs which is not directly related to total energy values. Table III lists the obtained vertical IPs with our practical NOF using EKT together with Koopmans' theorem (KT) IPs and experimental values. All values were calculated at experimental geometries of neutral molecules given in Ref. [90].

\section{[ TABLE III ]}

KT states that the IP is given by the HF orbital energy with opposite sign $\left(-\varepsilon_{i}\right)$, calculated in the neutral system. It has long recognized that in general there is an excellent agreement between the KT values and the experimental ones because of the fortuitous cancellation of the correlation and orbital relaxation effects. A survey of Table III reveals that KT consistently overestimates the first IPs except for $\mathrm{P}_{2}$.

The prevailing trend is that the values decrease in moving from KT to NOF-EKT, and then from NOF-EKT to experimental data. Conversely, in case of $\mathrm{P}_{2}$, the first IP increases in moving from KT to NOF-EKT, and then from NOF-EKT to experimental value. For $\mathrm{HCl}$ molecule, the NOF-EKT first IP is smaller than KT and experimental values. Generally, the NOF-EKT first IPs move closer to experimental data.

Table III lists also higher IPs calculated via KT and NOF-EKT methods. In general, the NOF-EKT and KT results are systematically larger than the experimental values. The behavior of higher IPs is quite similar to that for the first IP results, discussed previously. For most molecular orbitals, NOF-EKT values are smaller than $\mathrm{KT}$ and greater than the experimental data. There are molecular orbitals, for example, orbital $\pi$ for $\mathrm{CO}$ and orbital $b_{1}$ for $\mathrm{H}_{2} \mathrm{CO}$, with NOF-EKT values greater than KT and the experimental data. The exception is $\mathrm{P}_{2}$, for which the NOF-EKT $\sigma_{g}$ IP is decreased. The agreement of NOF-EKT and KT IPs with the experimental values is less precise for inner valence molecular orbitals, but again the NOF-EKT IPs move closer to experimental data.

An Important case is the $\mathrm{N}_{2}$ molecule. It is well-known that the $\mathrm{KT} \sigma_{g}$ and $\pi_{u}$ IPs are in the wrong order for this molecule. NOF-EKT calculations on $\mathrm{N}_{2}$ give valence shell IPs which are in correct order, and in general a numerical improvement is obtained over KT IPs. In case of the orbital $\pi_{u}$ for $\mathrm{N}_{2}$, the 
xxxii

KT IPs is closer to experimental data but due to the mentioned wrong ordering. The results are in good agreement with the corresponding experimental vertical IPs considering the small basis sets used for these calculations.

Electron affinities (EAs) are considerably more difficult to calculate than IPs. For example, EAs are much more sensitive to the basis set than the corresponding IPs. The EKT provides also the means for calculation of these magnitudes, but unfortunately EKT-EA description is often very poor. On the other hand, vertical EAs can be calculated by the energy difference for neutral molecules $\left(\mathrm{M}^{0}\right)$ and negative ions $\left(\mathrm{M}^{-}\right): E\left(\mathrm{M}^{0}\right)-E\left(\mathrm{M}^{-}\right)$at nearexperimental geometries of $\mathrm{M}^{0}$. Table IV lists the obtained vertical EAs for selected open-shell molecules.

\section{[ TABLE IV ]}

As can be seen, generally all electron affinities predicted by $\triangle \mathrm{SCF}$ are negative, indicating a more stable neutral system with respect to the anion. The inclusion of correlation via $\operatorname{CCSD}(\mathrm{T})$ and NOF approximates them to the available adiabatic experimental EAs, accordingly with the expected trend. The EAs tend to increase in moving from $\triangle \mathrm{CCSD}(\mathrm{T})$ to $\triangle \mathrm{NOF}$ and then from $\triangle \mathrm{NOF}$ to the experiment. It should be noted that $\mathrm{NH}$ anion is predicted to be unbound by $\operatorname{CCSD}(\mathrm{T})$, whereas the positive vertical EA value via NOF corresponds to bound anionic state.

For $\mathrm{OH}$ and $\mathrm{SH}$, the NOF EAs are larger than the experimental values. This trend is due to the expected underestimation of the correlation energy for open-shell states within our approach. In fact, we fix the unpaired electron in the corresponding HF higher-occupied molecular orbital (HOMO) of the neutral molecule, and then this level does not participate in the correlation. Note that for these molecules the total spin of the neutral molecule is greater than the total spin of the anion $\left(S_{\mathrm{N}}>S_{\mathrm{A}}\right)$. The underestimation of the total energy is for neutral molecules larger than for anions and therefore the NOF vertical EAs are overestimated.

Comparison with experimental results show that vertical NOF-EAs are better than those predicted by the $\operatorname{CCSD}(\mathrm{T})$ method within the $6-31++\mathrm{G}^{* *}$ basis set.

\subsubsection{Dipole Moment}

The dipole moments (DMs), different from zero, obtained using HF, CCSD(T) and NOF methods are presented in Table V. For comparison, we have also included into this Table the available experimental data [90].

\section{[ TABLE V ]}

The correlated dipole moments tend to be lower compared to HF DMs. An exception to this trend is noticed in the case of $\mathrm{CH}_{2}$, where the $\operatorname{CCSD}(\mathrm{T})$ $\mathrm{DM}$ is predicted to be higher than the HF value. Important cases are the CO 
and $\mathrm{N}_{2} \mathrm{O}$ molecules for which the $\mathrm{HF}$ approximation gives a DM in the wrong direction, whereas correlation methods approach it to the experimental value. The quality of the NOs is critical for the accuracy of NOF theory. For example, in the case of $\mathrm{CO}$ molecule, we achieved the sign inversion of the DM after improving further the basis sets. The DMs obtained with the NOF method are in good agreement with the available experimental data considering the basis sets $\left(6-31 \mathrm{G}^{* *}\right)$ used for these calculations.

\subsubsection{Equilibrium geometries and vibrational frequencies}

We have employed the non-gradient geometry optimization to determine the equilibrium bond distances $\left(r_{e}\right)$. For each molecule, we have calculated the total energy $U(r)$ at a dense grid of bond distances $r$, separated from each other by $10^{-3} \AA$. The harmonic vibrational frequencies $\left(\omega_{e}\right)$ were determined from the second derivatives of the energy with respect to the nuclear positions. The equilibrium force constants $k_{e}=U^{\prime \prime}\left(r_{e}\right)$ were obtained from least squares fits of the energy to a second-order polynomial in the distances,

$$
U(r)=U\left(r_{e}\right)+\frac{1}{2} k_{e}\left(r-r_{e}\right)^{2}
$$

Table VI lists the equilibrium geometries for the selected closed-shell diatomic molecules. It has long been recognized that the HF method gives reduced bond lengths. From Table VI we see that the correlated bond lengths are mostly longer than the experimental values. Exceptions are the bond distances predicted by $\mathrm{CCD}$ and $\mathrm{NOF}$ methods for $\mathrm{HCl}$ molecule.

\section{[ TABLE VI ]}

The values of the harmonic vibrational frequencies for the selected set of molecules are presented in Table VII. The expected trends of this property with respect to experiment are well reproduced. The HF results are systematically larger and the correlated vibrational frequencies move closer to experimental data. By both correlated methods, the experimental frequencies are still overestimated, except for the $\mathrm{HCl}$ molecule, for which the $\mathrm{NOF}$ frequency is lower than the experimental value.

\section{[ TABLE VII ]}

The performance of our practical NOF to predict equilibrium bond distances and vibrational frequencies is similar to CCD. 
xxxiv

TABLE I. Closed-shell total energies in Hartrees. (6-31G**)

\begin{tabular}{ccccc}
\hline Molecule & $\mathrm{HF}^{a}$ & $\mathrm{CCSD}(\mathrm{T})^{b}$ & $\mathrm{NOF}^{c}$ & B3LYP $^{d}$ \\
\hline $\mathrm{FH}$ & -100.009834 & -100.198698 & -100.178202 & -100.425817 \\
$\mathrm{H}_{2} \mathrm{O}$ & -76.022615 & -76.228954 & -76.207940 & -76.417892 \\
$\mathrm{NH}_{3}$ & -56.194962 & -56.399981 & -56.378970 & -56.556343 \\
$\mathrm{CO}$ & -112.736756 & -113.032821 & -113.020010 & -113.306694 \\
$\mathrm{HNO}$ & -129.783338 & -130.149630 & -130.185528 & -130.467334 \\
$\mathrm{H}_{2} \mathrm{CO}$ & -113.867947 & -114.202886 & -114.208902 & -114.500848 \\
$\mathrm{HCl}$ & -460.066040 & -460.224196 & -460.262033 & -460.800097 \\
$\mathrm{PH}$ & -342.452229 & -342.605974 & -342.635555 & -343.142663 \\
$\mathrm{BeS}$ & -412.103398 & -412.284575 & -412.310091 & -412.894462 \\
$\mathrm{~N}_{2} \mathrm{O}$ & -183.675227 & -184.203202 & -184.233374 & -184.656028 \\
$\mathrm{O}_{3}$ & -224.242821 & -224.867269 & -224.992524 & -225.400708 \\
$\mathrm{NaCl}$ & -621.397562 & -621.546221 & -621.586299 & -622.556987 \\
$\mathrm{P}_{2}$ & -681.421021 & -681.667624 & -681.742405 & -682.683866 \\
$\mathrm{SO}_{2}$ & -547.165010 & -547.686081 & -547.805959 & -548.579504 \\
$\mathrm{Cl}_{2}$ & -918.908639 & -919.198123 & -919.345443 & -920.341830 \\
\hline
\end{tabular}

${ }^{a}$ Hartree-Fock total energies.

${ }^{b} \operatorname{CCSD}(\mathrm{T})$ total energies.

${ }^{c}$ Natural Orbital Functional total energies.

${ }^{d}$ B3LYP total energies. 
RESULTS XXXv

TABLE II. Open-shell total energies in Hartrees. $\left(6-31++\mathrm{G}^{* *}\right)$

\begin{tabular}{ccccc}
\hline Molecule & $\mathrm{HF}^{a}$ & $\mathrm{CCSD}(\mathrm{T})^{b}$ & $\mathrm{NOF}^{c}$ & $\mathrm{~B}^{b} \mathrm{LYP}^{d}$ \\
\hline $\mathrm{CH}_{2}$ & -38.919309 & -39.037939 & -38.981051 & -39.158525 \\
$\mathrm{NH}$ & -54.957745 & -55.091649 & -55.017865 & -55.227209 \\
$\mathrm{OH}$ & -75.388827 & -75.555805 & -75.501346 & -75.739015 \\
$\mathrm{MgH}$ & -200.135519 & -200.169572 & -200.163233 & -200.632922 \\
$\mathrm{SH}$ & -398.064176 & -398.195038 & -398.192977 & -398.745129 \\
\hline
\end{tabular}

${ }^{a}$ Hartree-Fock total energies.

${ }^{b} \operatorname{CCSD}(\mathrm{T})$ total energies.

${ }^{c}$ Natural Orbital Functional total energies.

${ }^{d}$ B3LYP total energies. 
xxxvi

TABLE III. Vertical ionization potentials in eV. (6-31G**)

\begin{tabular}{ccccc}
\hline Molecule & $\mathrm{MO}$ & $\mathrm{KT}^{a}$ & NOF-EKT $^{b}$ & Exp $^{c}$ \\
\hline $\mathrm{FH}$ & $\pi$ & 17.06 & 16.82 & 16.19 \\
& $\sigma$ & 20.23 & 19.99 & 19.90 \\
$\mathrm{H}_{2} \mathrm{O}$ & $b_{1}$ & 13.53 & 13.08 & 12.78 \\
& $a_{1}$ & 15.56 & 15.54 & 14.83 \\
& $b_{2}$ & 19.10 & 18.93 & 18.72 \\
$\mathrm{NH}_{3}$ & $a_{1}$ & 11.44 & 11.05 & 10.80 \\
& $e$ & 16.87 & 16.93 & 16.80 \\
$\mathrm{CO}$ & $\sigma$ & 14.90 & 14.59 & 14.01 \\
& $\pi$ & 17.22 & 17.28 & 16.85 \\
& $\sigma$ & 21.67 & 21.58 & 19.78 \\
$\mathrm{~N}_{2}$ & $\sigma_{g}$ & 17.13 & 16.74 & 15.60 \\
& $\pi_{u}$ & 16.63 & 17.14 & 16.68 \\
$\mathrm{H}_{2} \mathrm{CO}$ & $\sigma_{u}$ & 21.11 & 20.96 & 18.78 \\
& $b_{2}$ & 11.87 & 11.54 & 10.90 \\
& $b_{1}$ & 14.46 & 14.63 & 14.50 \\
& $a_{1}$ & 17.58 & 17.44 & 16.10 \\
$\mathrm{HCl}$ & $b_{2}$ & 18.72 & 18.40 & 17.00 \\
& $\pi$ & 12.93 & 12.51 & $12.77^{d}$ \\
& $\sigma$ & 17.00 & 16.73 & $16.60^{d}$ \\
$\mathrm{P}_{2}$ & $\sigma$ & 30.42 & 26.57 & $25.80^{d}$ \\
& $\pi_{u}$ & 10.14 & 10.39 & $10.65^{e}$ \\
& $\sigma_{g}$ & 11.14 & 10.63 & $10.84^{e}$ \\
\hline & & & & \\
\hline
\end{tabular}

${ }^{a}-\varepsilon_{i}^{H F}$.

${ }^{b}$ Natural orbital functional vertical ionization potentials obtained from the extended Koopmans' theorem.

${ }^{c}$ Experimental vertical ionization potentials from Ref. [91].

${ }^{d}$ Experimental vertical ionization potential for $\mathrm{HCl}$ from Ref. [92].

${ }^{e}$ Experimental vertical ionization potential for $\mathrm{P}_{2}$ from Ref. [93]. 
RESULTS xxxvii

TABLE IV. Vertical electron affinities in eV. (6-31++ $\left.\mathrm{G}^{* *}\right)$

\begin{tabular}{ccccccc}
\hline Molecule & $S_{\mathrm{N}}^{a}$ & $S_{\mathrm{A}}^{b}$ & $\Delta \mathrm{SCF}^{c}$ & $\Delta \mathrm{CCSD}(\mathrm{T})^{d}$ & $\Delta \mathrm{NOF}^{e}$ & $\operatorname{Exp}^{f}$ \\
\hline $\mathrm{CH}_{2}$ & 1 & $\frac{1}{2}$ & -3.577 & -2.028 & -0.091 & $0.652(0.006)$ \\
$\mathrm{NH}$ & 1 & $\frac{1}{2}$ & -1.439 & -0.219 & 0.113 & $0.370(0.004)$ \\
$\mathrm{OH}$ & $\frac{1}{2}$ & 0 & -0.147 & 1.360 & 1.830 & $1.828(0.001)$ \\
$\mathrm{MgH}$ & $\frac{1}{2}$ & 0 & -0.057 & 0.612 & 0.976 & $1.05(0.06)$ \\
$\mathrm{SH}$ & $\frac{1}{2}$ & 0 & 1.246 & 1.774 & 2.761 & $2.314(0.003)$ \\
\hline
\end{tabular}

${ }^{a} S_{\mathrm{N}}$ : total spin of the neutral molecule.

${ }^{b} S_{\mathrm{A}}$ : total spin of the anion.

${ }^{c} \Delta \mathrm{SCF}=E_{\mathrm{HF}}\left(\mathrm{M}^{0}\right)-E_{\mathrm{HF}}\left(\mathrm{M}^{-}\right)$.

${ }^{d} \Delta \operatorname{CCSD}(\mathrm{T})=E_{\mathrm{CCSD}(\mathrm{T})}\left(\mathrm{M}^{0}\right)-E_{\mathrm{CCSD}(\mathrm{T})}\left(\mathrm{M}^{-}\right)$.

e $\Delta \mathrm{NOF}=E_{\mathrm{NOF}}\left(\mathrm{M}^{0}\right)-E_{\mathrm{NOF}}\left(\mathrm{M}^{-}\right)$.

${ }^{f}$ Experimental adiabatic electron affinities from Ref. [90]. The uncertainty is shown in parentheses. 
xxxviii

TABLE V. Dipole moments in Debyes. (6-31G**)

\begin{tabular}{ccccc}
\hline Molecule & $\mathrm{HF}^{a}$ & $\mathrm{CCSD}(\mathrm{T})^{b}$ & $\mathrm{NOF}^{c}$ & $\operatorname{Exp}^{d}$ \\
\hline $\mathrm{CH}_{2}$ & 0.54 & 0.57 & 0.51 & - \\
$\mathrm{NH}$ & 1.73 & 1.61 & 1.62 & $1.39^{f}$ \\
$\mathrm{OH}$ & 1.88 & 1.77 & 1.77 & 1.66 \\
$\mathrm{FH}$ & 1.98 & 1.87 & 1.84 & 1.82 \\
$\mathrm{H}_{2} \mathrm{O}$ & 2.20 & 2.09 & 2.08 & 1.85 \\
$\mathrm{NH}_{3}$ & 1.89 & 1.81 & 1.76 & 1.47 \\
$\mathrm{CO}$ & $0.33^{*}$ & 0.07 & $0.03^{e}$ & 0.11 \\
$\mathrm{MgH}$ & 1.41 & 1.10 & 1.22 & - \\
$\mathrm{SH}$ & 1.07 & 0.97 & 0.91 & 0.76 \\
$\mathrm{HCl}$ & 1.48 & 1.37 & 1.30 & 1.08 \\
$\mathrm{HNO}$ & 2.02 & 1.63 & 1.61 & 1.67 \\
$\mathrm{H}_{2} \mathrm{CO}$ & 2.75 & 2.18 & 2.36 & 2.33 \\
$\mathrm{PH}$ & 0.80 & 0.80 & 0.60 & 0.58 \\
$\mathrm{BeS}$ & 6.42 & 4.59 & 5.38 & - \\
$\mathrm{N}_{2} \mathrm{O}$ & $0.60^{*}$ & $0.07^{*}$ & 0.03 & 0.17 \\
$\mathrm{O}_{3}$ & 0.78 & 0.49 & 0.50 & 0.53 \\
$\mathrm{NaCl}$ & 9.39 & 8.97 & 9.03 & 9.0 \\
$\mathrm{SO}_{2}$ & 2.21 & 1.78 & 1.56 & 1.63 \\
\hline
\end{tabular}

* This value has an opposite sign relative to the experimental value

${ }^{a}$ Hartree-Fock dipole moments.

${ }^{b} \mathrm{CCSD}(\mathrm{T})$ dipole moments.

${ }^{c}$ Natural Orbital Functional dipole moments.

${ }^{d}$ Experimental dipole moments from Ref. [90].

$e$ This value was obtained with $6-311 \mathrm{G}^{* *}$

${ }^{f}$ Experimental dipole moment for NH from Ref. [94]. 
RESULTS xxxix

TABLE VI. Equilibrium bond lengths in $\AA$. (6-31G**)

\begin{tabular}{ccccc}
\hline Molecule & $\mathrm{HF}^{a}$ & $\mathrm{CCD}^{b}$ & $\mathrm{NOF}^{c}$ & $\operatorname{Exp}^{d}$ \\
\hline $\mathrm{FH}$ & 0.900 & 0.920 & 0.917 & 0.917 \\
$\mathrm{CO}$ & 1.114 & 1.139 & 1.132 & 1.128 \\
$\mathrm{HCl}$ & 1.265 & 1.271 & 1.273 & 1.275 \\
$\mathrm{BeS}$ & 1.733 & 1.744 & 1.746 & 1.742 \\
$\mathrm{~N}_{2}$ & 1.078 & 1.112 & 1.099 & 1.098 \\
$\mathrm{P}_{2}$ & 1.859 & 1.905 & 1.893 & 1.893 \\
\hline
\end{tabular}

${ }^{a}$ Hartree-Fock equilibrium bond lengths.

${ }^{b} \mathrm{CCD}$ equilibrium bond lengths.

${ }^{c}$ Natural Orbital Functional equilibrium bond lengths.

${ }^{d}$ Experimental equilibrium bond lengths from Ref. [90]. 
TABLE VII. Vibrational frequencies in $\mathrm{cm}^{-1}$. (6-31G**)

\begin{tabular}{ccccc}
\hline Molecule & $\mathrm{HF}^{a}$ & $\mathrm{CCD}^{b}$ & $\mathrm{NOF}^{c}$ & $\operatorname{Exp}^{d}$ \\
\hline $\mathrm{FH}$ & 4500 & 4213 & 4216 & 4138 \\
$\mathrm{CO}$ & 2442 & 2251 & 2284 & 2170 \\
$\mathrm{HCl}$ & 3182 & 3089 & 2927 & 2991 \\
$\mathrm{BeS}$ & 1069 & 1045 & 1047 & 998 \\
$\mathrm{~N}_{2}$ & 2761 & 2433 & 2544 & 2359 \\
$\mathrm{P}_{2}$ & 909 & 804 & 814 & 781 \\
\hline
\end{tabular}

${ }^{a}$ Hartree-Fock vibrational frequencies.

${ }^{b}$ CCD vibrational frequencies.

${ }^{c}$ Natural Orbital Functional vibrational frequencies.

${ }^{d}$ Experimental vibrational frequencies from Ref. [90] 


\subsection{CONCLUDING REMARKS}

${ }^{1} \mathbf{D}$-functional theory, explicitly given in terms of natural orbitals and their occupation numbers, has emerged as an alternative method to conventional approaches for considering the electronic correlation. This chapter has introduced important basic concepts for understanding the NOF formalism. We have also offered a brief characterization of almost all references concerning this theory published hitherto.

A free-parameter functional based on a new approach for the two-electron cumulant has been reviewed. This functional reduces to the exact expression for the total energy in two-electron systems and to the HF energy for idempotent 1-RDMs. Moreover, it is derived from a rigorous $N$-representable ansatz for the 2-RDM.

The mean value theorem provides a prescription for deriving a practical NOF that yields reasonable correlation energies for molecules. Accurate results for closed- and open-shell systems are obtained with energy expressions that only include two-index two-electron integrals. We shall improve this functional by providing a better approximation for the mean value $J_{i}^{*}$ of the Coulomb interactions. It is highly desirable to develop our restricted openshell formulation by appropriately expressing the 2-RDM in two-electron spin functions which are simultaneously eigenfunctions of $\widehat{S}_{z}$ and $\widehat{S}^{2}$.

The explicit form derived by Pernal for the effective nonlocal potential allows to establish one-electron equations that may be of great value for the development of efficient computational methods in NOF theory. Although recent progress has been made, NOF theory needs to continue its assessment. Some other essential conditions such as the reproduction of the homogeneous electron gas should be utilized in the evaluation of approximate implementations.

\section{Acknowledgement}

The author is grateful to Prof. Jesus M. Ugalde, Prof. Eduardo V. Ludeña, and Dr. Xabier Lopez for helpful discussions and comments. 



\section{References}

1. Coleman, A.J. Rev. Mod. Phys. 1963, 35, 668.

2. Hohenberg, P; Kohn, W. Phys. Rev. B 1964, 136, 846.

3. Parr, R.G.; Yang, W. Density-Functional Theory of Atoms and Molecules, Oxford University Press: Oxford, 1989; Kryachko, E.S.; Ludeña, E.V.; Energy Density Functional Theory of Many-Electron Systems, Kluwer: Dordrecht, 1990.

4. Kohn, W.; Sham, L.J. Phys. Rev. A 1965, 140, 1133.

5. Eschrig, H. The Fundamentals of Density Functional Theory; Teubner: Stuttgart, Germany, 1996.

6. Ludeña, E.V.; Karasiev, V.; Artemiev, A.; Gómez, D. In: Cioslowski, J.; Ed. Many-Electron Densities and Reduced Density Matrices, Kluwer: New York, 2000, p. 209.

7. Jarzęcki, A.A.; Davidson, E.R. Phys. Rev. A 1998, 58, 1902.

8. Tao, J; Perdew, J.P.; Staroverov, V.N.; Scuseria, G.E. Phys. Rev. Lett. 2003, 91, 146401; Perdew, J.P.; Tao, J; Staroverov, V.N.; Scuseria, G.E. J. Chem. Phys. 2004, 120, 6898.

9. Morrison, R.C.; Bartolotti, L.J. J. Chem. Phys. 2004, 121, 12151.

10. Levy, M. Proc. Natl. Acad. Sci. U.S.A. 1979, 76, 6062.

11. Levy, M. In: Erdahl, R.; Smith, V.H., Jr.; Eds. Density Matrices and Density Functionals, Reidel: Dordrecht, 1987, p. 479.

12. Cioslowski, J.; Pernal K.; Ziesche, P. J. Chem. Phys. 2002, 117, 9560;

13. Cioslowski, J. J. Chem. Phys. 2005, 123, 164106.

14. Levy, M; Perdew, J.P. Phys. Rev. A 1985, 32, 2010.

15. Gilbert, T.L. Phys. Rev. B 1975, 12, 2111.

16. Berrondo, M; Goscinski, O. Int J. Quantum Chem. 1975, 9S, 67. 
17. Donnelly, R.A.; Parr, R.G. J. Chem. Phys. 1978, 69, 4431.

18. Donnelly, R.A. J. Chem. Phys. 1979, 71, 2874.

19. Valone, S.M. J. Chem. Phys. 1980, 73, 1344.

20. Lieb, E.H. Phys. Rev. Lett. 1981, 46, 457.

21. Lieb, E.H. Int J. Quantum Chem. 1983, 24, 243.

22. Müller, A.M.K. Phys. Lett. A 1984, 105, 446.

23. Buijse, M.A. Ph.D. thesis, Vrije Universiteit Amsterdam, Amsterdam 1991.

24. Zumbach, G.; Maschke, K. J. Chem. Phys. 1985, 82, 5604.

25. Tung Nguyen-Dang, T.; Ludeña, E.V.; Tal, Y. J. Mol. Struct. (Theochem) 1985, 120, 247; Ludeña, E. V.; Sierraalta, A. Phys. Rev. A 1985, 32, 19; Ludeña, E. V. In: Erdahl, R.; Smith, V.H., Jr.; Eds. Density Matrices and Density Functionals, Reidel: Dordrecht, 1987, p. 289.

26. Redondo, A. Phys. Rev. A 1989, 39, 4366.

27. López-Sandoval, R.; Pastor, G.M. Phys. Rev. B 2000, 61, 1764.

28. Valdemoro, C. Phys. Rev. A 1992, 45, 4462.

29. Carlsson, A.E. Phys. Rev. B 1997, 56, 12058; Hennig, R.G.; Carlsson, A.E. Phys. Rev. B 2001, 63, 115116.

30. Klein, A.; Dreizler, R.M. Phys. Rev. A 1998, 57, 2485.

31. Cioslowski, J.; Lopez-Boada, R. J. Chem. Phys. 1998, 109, 4156; Chem. Phys. Lett. 1999, 307, 445.

32. Smith, D.W.; Day, O.W. J. Chem. Phys. 1975, 62, 113; Day, O.W.; Smith, D.W.; Garrot, C. Int. J. Quantum Chem. Symp. 1974, 8, 501.

33. Morrell, M.M.; Parr, R.G.; Levy, M. J. Chem. Phys. 1975, 62, 549.

34. Nooijen, M. J. Chem. Phys. 1999, 111, 8356.

35. Goedecker, S.; Umrigar, C.J. Phys. Rev. Lett. 1998, 81, 866.

36. Baerends, E.J. Phys. Rev. Lett. 2001, 87, 133004; Buijse, M.A.; Baerends, E.J. Mol. Phys. 2002, 100, 401.

37. Holas, A. Phys. Rev. A 1999, 59, 3454.

38. Cioslowski, J.; Pernal, K. J. Chem. Phys. 1999, 111, 3396. 
39. Cioslowski, J.; Pernal, K. Phys. Rev. A 2000, 61, 34503; Cioslowski, J.; Ziesche, P; Pernal, K. Phys. Rev. B 2001, 63, 205105.

40. Csanyi, G.; Arias, T.A. Phys. Rev. B 2000, 61, 7348.

41. Cioslowski, J.; Pernal, K. J. Chem. Phys. 2001, 115, 5784.

42. Mazziotti, D.A. J. Chem. Phys. 2000, 112, 10125.

43. Mazziotti, D.A. Chem. Phys. Lett. 2001, 338, 323.

44. Cohen, A.J.; Baerends, E.J. Chem. Phys. Lett. 2002, 364, 409.

45. Staroverov, V.N.; Scuseria, G.E. J. Chem. Phys. 2002, 117, 2489.

46. Csanyi, G.; Goedecker, S.; Arias, T.A. Phys. Rev. A 2002, 65, 32510.

47. Herbert, J.M.; Harriman, J.E. Int. J. Quantum Chem. 2002, 90, 355; J. Chem. Phys. 2003, 118, 10835; Chem. Phys. Lett. 2003, 382, 142.

48. Staroverov, V.N.; Scuseria, G.E. J. Chem. Phys. 2002, 117, 11107.

49. Durand, P. Cah. Phys. 1967, 21, 285; Goscinski, O. Int. J. Quantum Chem., Quantum Chem. Symp. 1982, 16, 591.

50. Lukman, B.; Koller, J.; Borštnik; Ažman, A. Mol. Phys. 1970, 18, 857.

51. England, W.B. J. Phys. Chem. 1982, 86, 1204; Int. J. Quantum Chem. 1983, 23, 905; Int. J. Quantum Chem., Quantum Chem. Symp. 1983, 17, 357.

52. Piris, M.; Cruz, R. Int. J. Quantum Chem. 1995, 53, 353; Piris, M.; Montero, L.A.; Cruz, N. J. Chem. Phys. 1997, 107, 180; Piris, M. J. Math. Chem. 1998; 23, 399; Piris, M.; Otto, P. J. Chem. Phys. 2000, $1112,8187$.

53. Piris, M. J. Math. Chem. 1999; 25, 47.

54. Barbiellini, B.; Bansil, A. J. Phys. Chem. Solids 2001, 62, 2181.

55. Yasuda, K. Phys. Rev. A 2001, 63, 32517; Yasuda, K. Phys. Rev. Lett. $2002,88,53001$.

56. Cioslowski, J.; Ziesche, P.; Pernal, K. J. Chem. Phys. 2001, 115, 8725.

57. Cioslowski, J.; Pernal, K. J. Chem. Phys. 2001, 116, 4802; Cioslowski, J.; Pernal, K. J. Chem. Phys. 2002, 117, 67.

58. Piris, M.; Otto, P. Int J Quantum Chem 2003, 94, 317.

59. Piris, M.; Martinez, A.; Otto, P. Int J Quantum Chem 2004, 97, 827. 


\section{xlvi REFERENCES}

60. Leiva, P.; Piris, M. J. Mol. Struct. (Theochem) 2005, 719, 63.

61. Piris, M.; Otto, P. Int J Quantum Chem 2005, 102, 90.

62. Kollmar, C.; Hess, B.A. J. Chem. Phys. 2004, 120, 3158.

63. Lathiotakis, N.N.; Helbig, N.; Gross, E.K.U. Phys. Rev. A 2005, 72, 30501.

64. Alcoba, D.R.; Lain, L.; Torre, A.; Bochicchio, R.C. J. Chem. Phys. 2005, $123,144113$.

65. Cioslowski, J.; Pernal K.; Buchowiecki, M. J. Chem. Phys. 2003, 119, 6443.

66. Kollmar, C.; Hess, B.A. J. Chem. Phys. 2003, 119, 4655.

67. Cioslowski, J.; Buchowiecki, M.; Ziesche, P. J. Chem. Phys. 2003, 119, 11570 .

68. Pernal, K.; Cioslowski, J. J. Chem. Phys. 2004, 120, 5987.

69. Cioslowski, J.; Pernal, K. J. Chem. Phys. 2004, 120, 10364.

70. Kollmar, C. J. Chem. Phys. 2004, 121, 11581.

71. Gritsenko, O.; Pernal, K.; Baerends, E.J. J. Chem. Phys. 2005, 122, 204102.

72. Pernal, K.; Cioslowski, J. Chem. Phys. Lett. 2005, 412, 71.

73. Piris, M. Int. J. Quantum Chem. 2006, 106, 1093.

74. Mazziotti, D.A. Chem. Phys. Lett. 1998, 289, 419; Mazziotti, D. A. Int. J. Quantum Chem. 1998, 70, 557.

75. Kutzelnigg, W. ; Mukherjee, D. J. Chem. Phys. 1999, 110, 2800.

76. Goedecker, S.; Umrigar, C. J. In: Cioslowski, J.; ed. Many-Electron Densities and Reduced Density Matrices, Kluwer/Plenum: New York, 2000, 165.

77. Kutzelnigg, W. Theor. Chim. Acta 1963, 1, 327.

78. Leiva, P.; Piris, M. J. Theo. Comp. Chem. 2005, 4, 1165.

79. Leiva, P.; Piris, M. J. Chem. Phys. 2005, 123, 214102.

80. Leiva, P.; Piris, M. Int J Quantum Chem 2006 (in press).

81. Pernal, K. Phys. Rev. Lett. 2005, 94, 233002.

82. Löwdin, P.-O. Phys. Rev. 1955, 97, 1474. 
83. McWeeny, R.; Rev. Mod. Phys. 1960, 32, 335; McWeeny, R.; Mizuno, Y. Proc. R. Soc. London, Ser. A 1961, 259, 554.

84. Katriel, J. ; Davidson, E.R. Proc. Natl. Acad. Sci. USA 1980, 77, 4403.

85. Bingel, W.A.; Kutzelnigg, W. Adv. Quantum. Chem. 1970, 5, 201; Harriman, J.E. Int. J. Quantum Chem. 1977, 12, 1039.

86. Fletcher, R. Practical Methods of Optimization, 2nd Edition, John Wiley \& Sons: New York, 1987.

87. Hariharan, P.C.; Pople, J.A. Theoret. Chimica Acta 1973, 28, 213; Francl, M.M.; Petro, W.J.; Hehre, W.J.; Binkley, J.S.; Gordon, M.S.; DeFrees, D.J.; Pople, J.A. J. Chem. Phys. 1982, 77, 3654.

88. Levine, I. N. Quantum Chemistry, Prentice Hall: Upper Saddle River, New Jersey 07458, 2000.

89. Frisch, M.J. ; Trucks, G.W.; Schlegel, H.B.; Gill, P.M.W.; Johnson, B.G.; Robb, M.A.; Cheeseman, J.R.; Keith, T.; Petersson, G.A.; Montgomery, J.A.; Raghavachari, K.; Al-Laham, M.A.; Zakrzewski, V.G.; Ortiz, J.V.; Foresman, J. B.; Cioslowski, J.; Stefanov, B.B.; Nanayakkara, A.; Challacombe, M.; Peng, C.Y.; Ayala, P.Y.; Chen, W.; Wong, M.W.; Andres, J.L.; Replogle, E.S.; Gomperts, R.; Martin, R.L.; Fox, D.J.; Binkley, J.S.; Defrees, D.J.; Baker, J.; Stewart, J.P.; Head-Gordon, M.; Gonzalez, C.; Pople, J.A. GAUSSIAN 94 Inc. 1995, Pittsburgh PA.

90. Johnson III, R.D.; ed. NIST Computational Chemistry Comparison and Benchmark Database, NIST Standard Reference Database Number 101, Release 11, May 2005 (http://srdata.nist.gov/cccbdb).

91. Morrison, R.C. J. Chem. Phys. 1996, 96, 3718.

92. von Niessen, W.; Asbrink, L.; Bieri, G. J. Electron. Spectrosc. Relat. Phenom. 1982, 26, 173.

93. Potts, A.W.; Glenn, K.G.; Price, W.C. Discuss. Faraday Soc. 1972, 54, 65.

94. Oliphant, N.; Bartlett, R.J.J. Chem. Phys. 1994, 100, 6550. 